\title{
fzr-1 and lin-35/Rb function redundantly to control cell proliferation in $C$. elegans as revealed by a nonbiased synthetic screen
}

\author{
David S. Fay, ${ }^{1,2}$ Sean Keenan, and Min Han ${ }^{2}$ \\ Howard Hughes Medical Institute and Department of Molecular, Cellular, and Developmental Biology, University \\ of Colorado, Boulder, Colorado 80309-0347, USA
}

\begin{abstract}
We report here a synthetic-lethal screen in Caenorhabditis elegans that overcomes a number of obstacles associated with the analysis of functionally redundant genes. Using this approach, we have identified mutations that synthetically interact with lin-35/Rb, a SynMuv gene and the sole member of the Rb/pocket protein family in C. elegans. Unlike the original SynMuv screens, our approach is completely nonbiased and can theoretically be applied to any situation in which a mutation fails to produce a detectable phenotype. From this screen we have identified fzr-1, a gene that synthetically interacts with lin-35 to produce global defects in cell proliferation control. fzr-1 encodes the C. elegans homolog of Cdh1/Hct1/FZR, a gene product shown in other systems to regulate the APC cyclosome. We have also uncovered genetic interactions between fzr-1 and a subset of class B SynMuv genes, and between lin-35 and the putative SCF regulator lin-23. We propose that lin-35, fzr-1, and lin-23 function redundantly to control cell cycle progression through the regulation of cyclin levels.
\end{abstract}

[Key Words: lin-35; retinoblastoma; fizzy related; fzr-1; C. elegans; hyperproliferation; synthetic screen]

Received October 5, 2001; revised version accepted December 20, 2001.

Genetic redundancy is a common feature of all eukaryotes, ensuring both a high degree of regulatory control and protection against mutational assault. It is also an impediment to biologists seeking to determine gene function through forward and reverse genetic approaches. Mutations in functionally redundant genes may show no obvious phenotype on their own but, when present in specific combinations, can lead to severe defects (i.e., a synthetic phenotype). Therefore, the ability to assign functions to this class of genes based solely on the availability and analysis of single mutants may be difficult or impossible.

Estimates from yeast suggest that $\sim 40 \%$ of all genes may fail to show even weak phenotypes when deleted (Smith et al. 1996; Winzeler et al. 1999), whereas a recent large-scale functional analysis of Caenorhabditis elegans genes using RNAi methods failed to detect phenotypes for $>85 \%$ of the genes examined (Fraser et al. 2000). In addition, recent small-scale deletion studies as well as previous mutational analyses suggest that functional disruptions of most genes in C. elegans fail to produce

\footnotetext{
${ }^{1}$ Present address: Department of Molecular Biology, University of Wyoming, PO Box 3944, Laramie, WY 82071-3944, USA.

${ }^{2}$ Corresponding authors.

E-MAIL davidfay@uwyo.edu; FAX (307) 766-5098.

E-MAIL mhan@colorado.edu; FAX (303) 735-0175.

Article and publication are at http://www.genesdev.org/cgi/doi/10.1101/ gad.952302.
}

obvious phenotypic effects (for review, see Hodgkin 2001). This apparent lack of an observable gene function may be the result of limitations at the level of detection as well as in the methodology used in the case of RNAi analysis. However, it is also likely a consequence of functional overlap conferred by either structurally related proteins or by molecularly distinct but functionally connected pathways. Evidence from yeast suggests that this latter explanation may, in fact, be the more common cause of genetic redundancy (Winzeler et al. 1999; for review, see Tautz 2000; Wagner 2000).

Given the difficulties in detecting synthetic interactions, relatively few examples of such nonhomologous genetic redundancies have been well characterized in $C$. elegans (Culotti et al. 1981; Johnson et al. 1981; Ferguson and Horvitz 1989; Davies et al. 1999). The best-known case of functional redundancy in C. elegans is the synthetic multivulval (SynMuv) genes (Ferguson et al. 1987; Ferguson and Horvitz 1989; for review, see Fay and Han 2000). SynMuv genes act in opposition to Ras/Map kinase pathway signaling to prevent the adoption of ectopic vulval cell fates during postembryonic development (Ferguson et al. 1987; Lu and Horvitz 1998). The relatively straightforward identification of SynMuv genes was made feasible by the ease with which Muv animals can be detected, the normally rare incidence of Muv animals in mutagenized populations, and the fact that double mutants are generally viable. SynMuv genes are 
commonly assigned membership into one of two classes, A or B. Pairwise combinations of mutations in both a class A gene and a class B gene lead to the expression of the Muv phenotype (Ferguson and Horvitz 1989).

The past several years have seen the molecular identification of many SynMuv genes through both forward and reverse genetic approaches. Although some encode peptides with unknown cellular or biochemical functions, a sizable fraction of class B genes have been linked (based on sequence homology) to transcriptional repression mechanisms. These include multiple members of the NuRD (nucleosome remodeling and histone deacetylase) complex (Lu and Horvitz 1998; Solari and Ahringer 2000; von Zelewsky et al. 2000), E2F and its binding partner Dp (Ceol and Horvitz 2001), and the solitary C. elegans Rb/ pocket protein homolog LIN-35 (Lu and Horvitz 1998).

In dramatic contrast to $\mathrm{Rb}$ knockout mutations in flies and mice (Clarke et al. 1992; Jacks et al. 1992; Lee et al. 1992; Du and Dyson 1999; for review, see Lipinski and Jacks 1999), strong loss-of-function (LOF) mutations in lin-35 are not lethal and have relatively subtle effects on viability and development (Lu and Horvitz 1998; this paper). Thus, based on the SynMuv screens, the single function ascribed to lin-35 affected only a small number of postembryonic blast cells and, moreover, required the presence of a second-site mutation to be revealed. These observations and the finding that LIN-35 is expressed broadly throughout development (Lu and Horvitz 1998) led us to hypothesize that: (1) lin-35 must carry out additional functions during C. elegans development; and (2) the identification of such functions will necessitate the isolation of second-site mutations that interact synthetically with mutations in lin-35 to produce novel phenotypes.

Using a nonbiased genetic approach, we have isolated a set of mutations that interact synthetically with lin-35. We report here the molecular identification and phenotypic characterization of one of these mutants, $f z r-1$, that affects the function of the C. elegans homolog of Cdh1/ Hct1/Fizzy Related (FZR; Schwab et al. 1997; Sigrist and Lehner 1997; Visintin et al. 1997). In combination, mutations in $f z r-1$ and lin-35 lead to a striking hyperproliferation phenotype, showing for the first time that LIN35 functions globally to control cell proliferation in $C$. elegans. In addition, we have also identified a synthetic requirement for lin-35 and fzr-1 during embryonic development, and describe an additional synthetic interaction between lin-35 and the putative SCF complex (Skp1, Cullin, F-box) regulator, lin-23 (Kipreos et al. 2000).

\section{Results}

A targeted strategy to identify novel lin-35 synthetic mutations

As a starting point for identifying novel lin-35/Rb synthetic mutants, we engineered strain MH1461 with two key components. The strain is (1) homozygous for a strong LOF mutation at the endogenous lin-35 locus (n745) and (2) carries an extrachromosomal array (kuEx119) containing wild-type rescuing copies of lin-35 and a GFP reporter that is ubiquitously expressed (also see Materials and Methods). kuEx119 fails to be transmitted to $\sim 25 \%$ of progeny derived from $\mathrm{kuEx} 119^{+}$parents. Thus, $\mathrm{kuEx} 119^{+}$animals give rise to both $\mathrm{kuEx} 119^{+}$ $\left.\left(G F P^{+} / \text {lin-35 }\right)^{+}\right)$and $k u E x 119^{-}\left(G F P^{-} /\right.$lin35 $\left.5^{-}\right)$progeny (Figs. 1 and 2A,B).

MH1461 animals were mutagenized and an $\mathrm{F}_{2}$ clonal screen was carried out (also see Materials and Methods). Strains acquiring desirable mutations would be predicted to show clear-cut differences in the phenotypes of kuEx $119^{+}$and $k u E x 119^{-}$progeny in the $\mathrm{F}_{3}$ generation (Fig. 1). Through screening of $\sim 3500$ haploid genomes, we isolated seven mutations defining seven distinct loci. Animals homozygous for mutations in lin-35 and the individual slr (ynthetic with $\underline{\operatorname{lin}}-35 / \underline{\mathrm{R} b}$ ) genes show a range of phenotypes including reduced size, slow growth rates, larval lethality, and sterility (D.S. Fay and M. Han, unpubl.). As anticipated, the screen also uncovered a number of class A SynMuv mutations that were not further pursued. We describe here the phenotypic analysis and molecular identification of one lin-35 synthetic mutation, slr-1(ku298). Based on the molecular identity of the gene defined by this mutation (see below), we refer to this gene as $f z r-1$.

\section{Synthetic interaction between lin-35 and fzr-1}

In screening for lin-35 synthetic mutants, we isolated candidate strain MH1621. MH1621 animals that carry kuEx119 are healthy, fertile, and generally indistinguishable from wild-type animals. In contrast, kuEx119- animals derived from this strain are nonviable and show multiple defects (Fig. 2C-F; see below). Genetic analyses indicated that the mutant phenotype observed in $k u E x 119^{-}$progeny resulted from the combined effect of mutations in lin-35 and a second unlinked gene, $f_{z r}-1$.

As a further test for specificity, we inactivated lin-35 using RNAi methods in strain MH1621. As expected, lin-35(RNAi) led to the highly penetrant expression of the kuEx119- phenotype in kuEx119+ animals (Fig. $2 \mathrm{G}, \mathrm{H}$; see below), as a result of eliminating LIN-35 activity derived from the array. Based on these criteria, we conclude that the phenotype observed in MH1621 kuEx119- progeny (hereafter referred to as lin-35; fzr-1 double mutants) is the direct result of a synthetic interaction between lin-35 and $f z r-1$.

$f z r-1$ single mutants isolated from strain MH1621 are viable and show no obvious anatomical or behavioral defects (see below). We did observe a low level of sterility in $f z r-1$ hermaphrodites $(3 \%, n=104)$ as well as an $~ 50 \%$ reduction in brood size compared with wild type $(142 \pm 37, n=8$, vs. $264 \pm 18, n=50)$. In addition, $f z r-1$ hermaphrodites produce males at an elevated frequency compared with wild type $(1.74 \%, n=1205$, vs. $\sim 0.2 \%$; Sulston and Hodgkin 1988) and show an increase in the occurrence of embryonic lethality $(11.9 \%, n=1241$, vs. $<1 \%, n>1000)$. An examination of lin-35 single mutants revealed similar low levels of sterility $(4 \%, n=100)$ and embryonic lethality $(6 \%, n=382)$, as well as an $\sim 65 \%$ reduction in brood size $(98 \pm 43, n=10)$. 


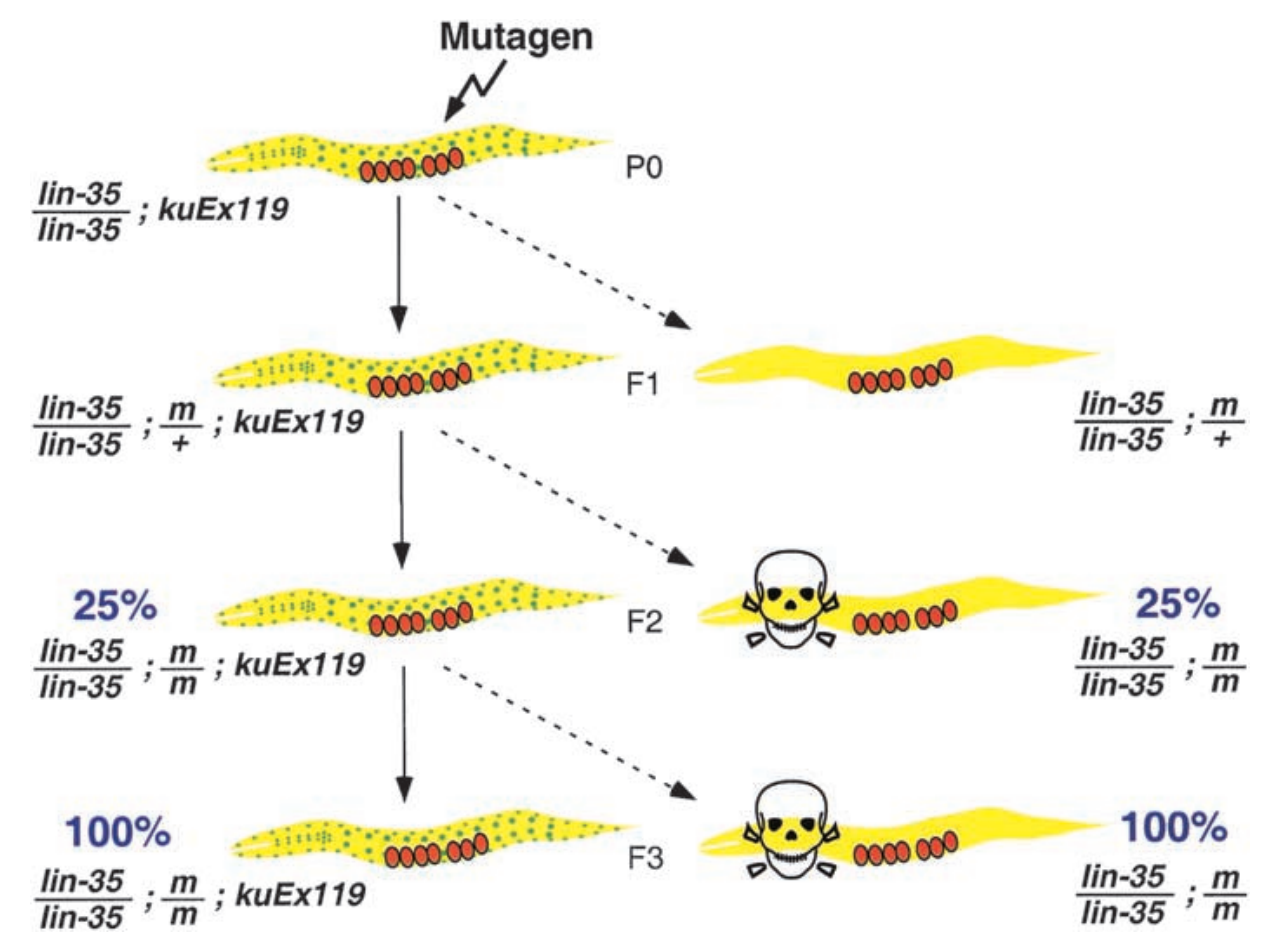

Figure 1. Outline of lin-35 synthetic screen. Following chemical mutagenesis, three consecutive generations of kuEx119+ animals are single-cloned and allowed to self-fertilize. Animals acquiring silent or otherwise viable mutations $(m)$ that show synergy with lin-35 are identified by the absence of wild-type-appearing kuEx $119^{-}$progeny in the final $\left(\mathrm{F}_{3}\right)$ generation. Only animals carrying mutations are shown in subsequent generations. For further details, see text.

Whereas only $12 \%(n=362)$ of $k u E x 119^{+}$animals derived from MH1621 fail to complete embryogenesis, $42 \%(n=98)$ of sibling lin-35; fzr-1 embryos fail to hatch, often arresting close to the end of elongation phase. For double mutants that successfully completed embryogenesis, the duration of embryonic development was similar to that observed for wild-type animals $(\sim 12-16 \mathrm{~h})$. In contrast, the length of postembryonic development was greatly extended in lin-35; fzr-1 animals, requiring 72 to $>96 \mathrm{~h}$ at $20^{\circ} \mathrm{C}(n=30)$ to complete larval stages versus 44 to $52 \mathrm{~h}(n=200)$ for wild-type animals (Fig. 2C,D; data not shown). Adult lin-35; fzr-1 animals show an approximate threefold reduction in total body volume compared with wild-type animals (Fig. 2E,F). In addition, some adult lin-35; $f z r-1$ hermaphrodites produce small numbers of nonviable embryos, which remain within the uterus and show pleiotropic defects (Fig. 2E; data not shown; also see below).

lin-35; fzr-1 double mutants show hyperproliferation in multiple tissue types

An examination of lin-35; $f z r-1$ double-mutant adults revealed extensive tissue hyperproliferation affecting subsets of cells derived from all the major germ layers (Sulston and Horvitz 1977). The supernumerary divisions appear to take place exclusively during postembryonic development as newly hatched lin-35; fzr-1 L1 larvae show no evidence of excess cell divisions and are pheno- typically indistinguishable from wild type (data not shown).

We measured the extent of hyperproliferation in three lineages derived from ectoderm in lin-35 and $f z r-1$ double mutants. Using a seam-cell-specific GFP marker (gift of J. Rothman, University of California, Santa Barbara), we observed up to a twofold increase in the number of hypodermal seam cells in lin-35; fzr-1 double mutants and in $f z r-1$ single mutants wherein lin-35 was inactivated by RNAi (Table 1). Interestingly, normal numbers of seam cells were observed in lin-35; fzr-1 double mutants derived from lin-35 heterozygous hermaphrodites, indicating that lin-35 maternal contributions are capable of suppressing hyperproliferation in at least some lineages (Table 1). Seam-cell proliferation was also normal in lin35 and $f z r-1$ single mutants (Table 1).

Hyperproliferation was also observed in vulval tissue (Table 1; Fig. 3B) as well as in cells that make up the somatic gonad (data not shown). An analysis of vulval development revealed complex and variable defects in both induction and cell division patterns. This was owing in part to the presence of extra vulval precursor cells (VPCs) that were present in L2/L3 animals and were competent to respond to the induction signal. Induction of these supernumerary VPCs appeared to account for most or all of the extra vulval cells observed. VPC induction in lin-35; fzr-1 mutants was dependent on lin-3 signal: 13/38 fzr-1; lin-3(e1417); lin-35(RNAi) animals failed to produce any vulval tissue, similar to results 


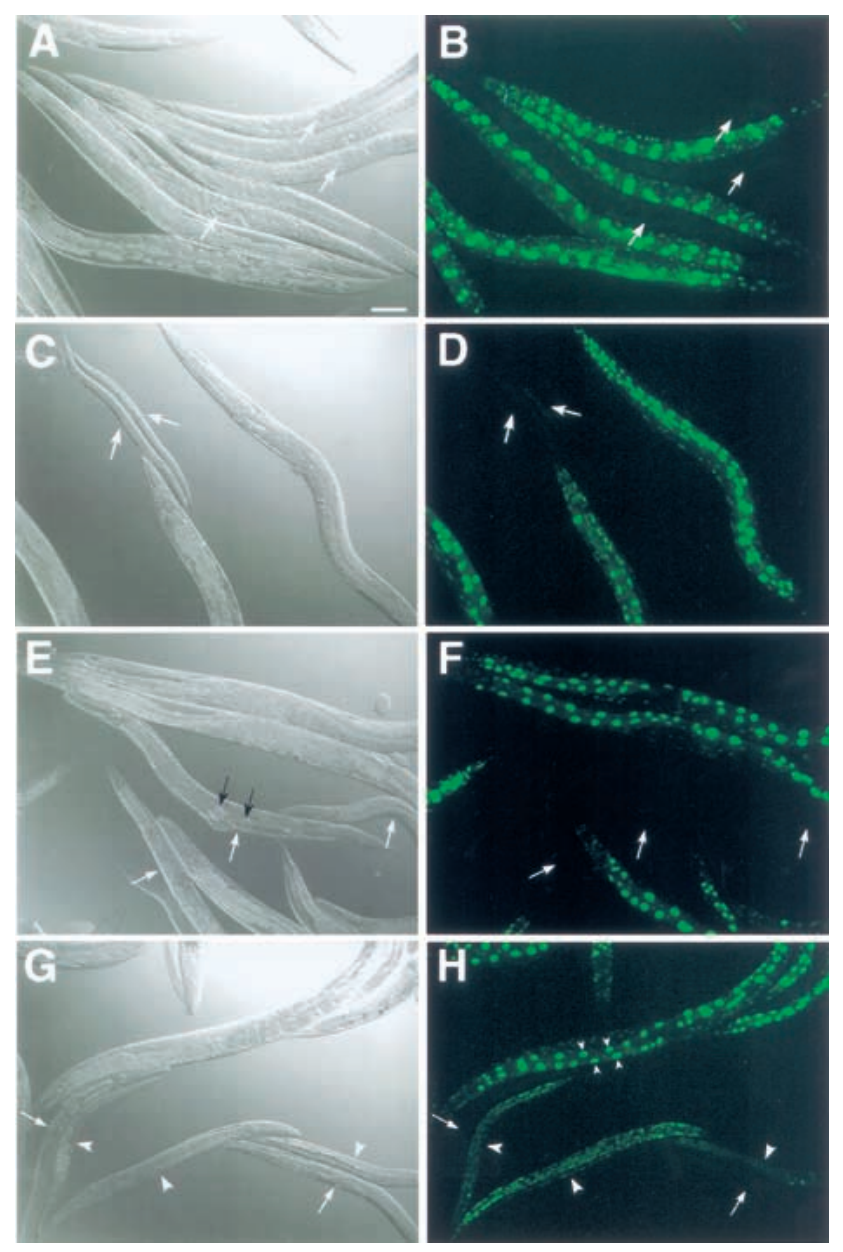

Figure 2. Synergism between lin-35 and fzr-1. $(A, C, E, G)$ DIC and corresponding $(B, D, F, H)$ GFP fluorescence images. White arrows indicate positions of kuEx119- animals. $(A, B)$ The starting strain for mutagenesis, MH1461 (Iin-35; kuEx119). Note the identical appearance of $\mathrm{kuEx} 119^{+/-}$adult animals. $(C-F)$ kuEx119+/- progeny from strain MH1791 (lin-35; fzr-1; kuEx119), $(C, D) \sim 60 \mathrm{~h}$ and $(E, F) \sim 96 \mathrm{~h}$ posthatching. Note pronounced developmental-stage $(C, D)$ and size $(E, F)$ differences between $\mathrm{kuEx} 119^{+/-}$animals. Black arrows indicate the location of nonviable embryos. (D) $\mathrm{F}_{1}$ progeny of MH1791 animals injected with lin-35 dsRNA $(1.2 \mu \mathrm{g} / \mu \mathrm{L})$. Large white arrowheads indicate the positions of $\mathrm{kuEx} 119^{+}$; lin-35(RNAi) affected animals; small white arrowheads, GFP-expressing nuclei in untreated $\mathrm{kuEx} 119^{+}$adults. Note the similar appearance of kuEx $119^{-}$animals to $k u E x 119^{+}$animals affected by RNAi. Also note the absence of large GFP-expressing intestinal cells in the kuEx119+ RNAi-affected animals. Size bar, $100 \mu \mathrm{m}$.

obtained for lin-3(e1417) mutants alone (10/21). Based on cell position assignments, the induced VPCs appeared to be derived exclusively from progenitor Pn.p cells that normally contribute to the vulva (P5.p, P6.p, and P7.p).

Following induction, VPCs underwent at least two to three rounds of divisions that were markedly slower and asynchronous compared with wild type. Whereas induced VPCs normally divide every $2 \mathrm{~h}$, we often observed division times of $>4 \mathrm{~h}$ and failed to observe accelerated division cycles. In addition, the pattern of vulval cell fates based on division plane orientations and cellfate-specific markers (lin-11::GFP) was highly variable (data not shown). These defects led to aberrant vulval morphologies; in some of these cases vulval cells failed to integrate into a single structure, producing multiple closely spaced invaginations reminiscent of a Muv phenotype (Fig. 3G,H).

In contrast to seam-cell and vulval lineages, ventral cord GABA neurons expressing unc-47::GFP (gift of E. Jorgensen, University of Utah, Salt Lake Cityl appeared to be completely unaffected in lin-35; $f z r-1$ double mutants (Table 1). This absence of a phenotype may be due to the lack of a requirement for lin-35 and $f z r-1$ in controlling proliferation in these cells, or may result from suppression by maternal products of $f z r-1$.

Cells derived from the mesoderm were also affected, including distal-tip cells (DTCs), which guide migration of gonad arms during postembryonic development. lin35 ; $f z r-1$ double mutants showed a high frequency of ex-

Table 1. Hyperproliferation of lin-35; fzr-1 double mutants

\begin{tabular}{|c|c|c|c|}
\hline & $\begin{array}{l}\text { Average } \\
\text { cell no. }\end{array}$ & Range & $n$ \\
\hline \multicolumn{4}{|c|}{ Hypodermal seam-cell nuclei ${ }^{a}$} \\
\hline Wild type & $15.9 \pm 1.2$ & $14-19$ & 25 \\
\hline $\operatorname{lin}-35$ & $16.3 \pm 0.9$ & $15-18$ & 8 \\
\hline$f z r-1$ & $16.5 \pm 1.1$ & $15-19$ & 25 \\
\hline fzr-1; 1 in-35(RNAi) & $21.2 \pm 3.5$ & $16-30$ & 30 \\
\hline $\operatorname{lin}-35^{m+} ; f z r-1^{m+}$ & $17.1 \pm 1.1$ & $16-19$ & 8 \\
\hline $\operatorname{lin}-35^{\mathrm{m}+} ; \mathrm{fzr}-1$ & $17.3 \pm 1.5$ & $16-21$ & 17 \\
\hline lin-35; $f z r-1^{m+}$ & $23.2 \pm 2.5$ & $18-26$ & 9 \\
\hline \multicolumn{4}{|l|}{ Vulval cell nuclei } \\
\hline Wild type & $22.0 \pm 0.0$ & & $>100$ \\
\hline lin-35 & $22.0 \pm 0.0$ & & 20 \\
\hline$f z r-1$ & $22.0 \pm 0.0$ & & 20 \\
\hline lin-35; fzr-1 & $30.6 \pm 5.8$ & $22-43$ & 13 \\
\hline \multicolumn{4}{|c|}{ unc-47::GFP-expressing GABA neurons ${ }^{\mathrm{b}}$} \\
\hline Wild type & $18.4 \pm 0.8$ & $17-19$ & 12 \\
\hline $\operatorname{lin}-35^{m+} ; f z r-1^{m+}$ & $18.9 \pm 0.8$ & $18-20$ & 12 \\
\hline $\operatorname{lin}-35^{m+} ; f z r-1$ & $18.4 \pm 0.7$ & $17-19$ & 7 \\
\hline lin-35; $f z r-1^{m+}$ & $18.1 \pm 0.9$ & $17-19$ & 10 \\
\hline fzr-1; lin-35(RNAi) & $18.5 \pm 0.7$ & $17-19$ & 20 \\
\hline \multicolumn{4}{|l|}{ Intestinal cell nuclei ${ }^{\mathrm{c}}$} \\
\hline Wild type & $26.3 \pm 0.4$ & $20-32$ & 43 \\
\hline$f_{z r-1}$ & $35.8 \pm 1.5$ & $23-48$ & 21 \\
\hline lin-35(RNAi) & $29.0 \pm 0.7$ & $20-39$ & 43 \\
\hline$f z r-1 ; \operatorname{lin}-35(R N A i)^{\mathrm{d}}$ & $52.2 \pm 7.1$ & $44-67$ & 20 \\
\hline
\end{tabular}

Cell numbers were scored for wild-type and mutant animals either directly (vulval cells) or using cell-type-specific integrated GFP markers.

${ }^{a}$ Hypodermal seam cells were scored using a seam-cell-specific GFP marker strain, JR632. $m+$ indicates the presence of maternal gene product.

bunc-47::GFP-expressing GABA neurons were assayed using a GFP marker strain, oxln12. Only those cells located between the rectum and pharyngeal grinder were scored.

'Intestinal cells were scored using an elt-2::GFP marker strain. ${ }^{\mathrm{d}}$ Owing to weak and variable GFP staining in $f z r-1$; lin35(RNAi) animals, numbers obtained are likely to be underestimates of the total number of intestinal cells (also see text). 


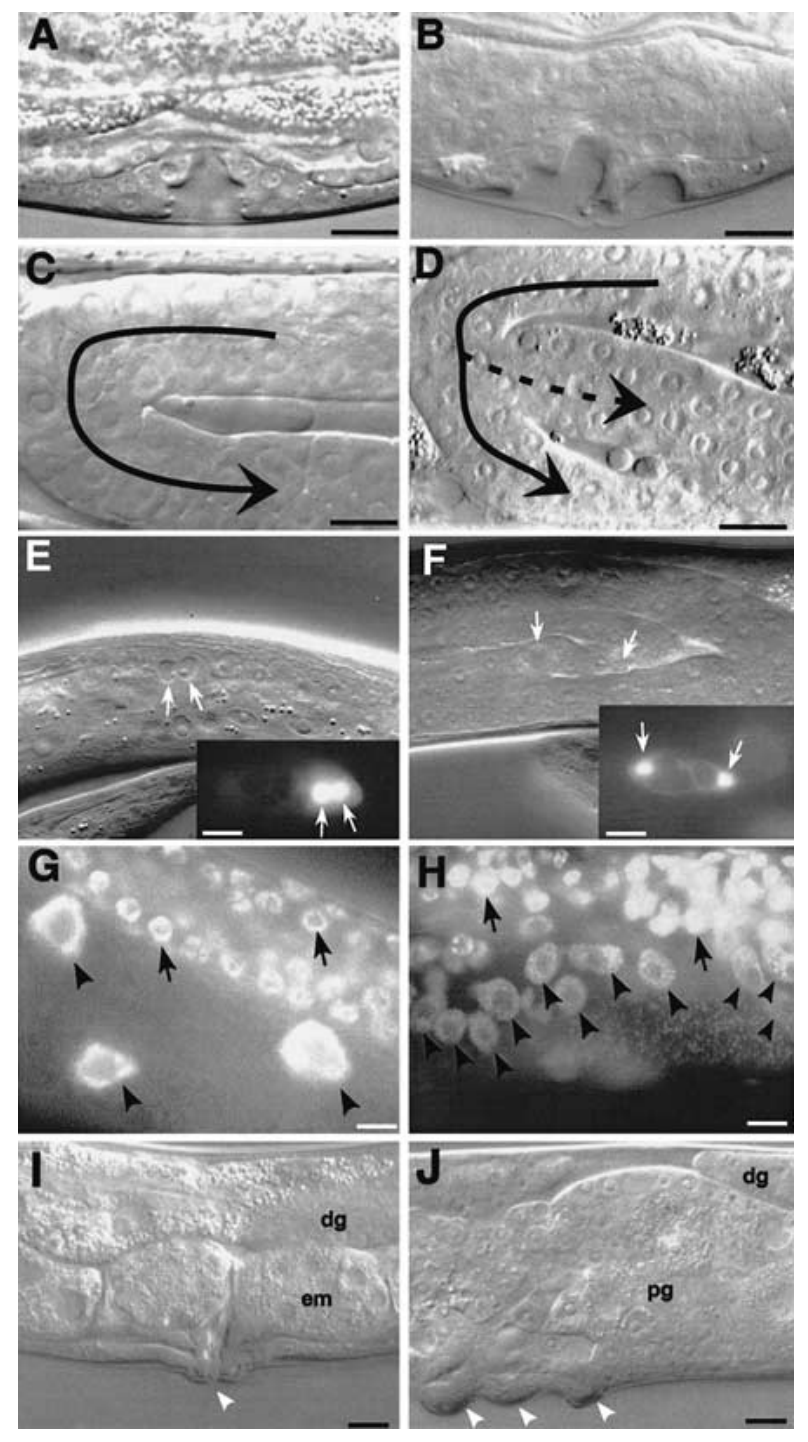

Figure 3. Hyperproliferation of lin-35; $f z r-1$ double mutants. $(A, C, G, I)$ lin-35; fzr-1; kuEx119 and $(B, D, H, J)$ lin-35; fzr-1 animals. $(A, B)$ L4-stage vulvae. The vulva in $A$ contains 22 nuclei and is indistinguishable from wild type, whereas the vulva in $B$ contains 32 nuclei and shows an abnormal morphology. $(C, D)$ Morphology of the gonad arm in L4/young-adult animals. The gonad arm in $C$ appears completely wild type whereas that in $D$ contains a single bifurcation, the result of an extra division of the distal tip cell. $(E, F)$ Distal tip cells (arrows) in $f z r-1$; lin35(RNAi) animals expressing lag-2::GFP. The DTCs in $E$ are in the process of dividing whereas $F$ shows cells several hours after divisions have taken place. The images in $E$ and $F$ are of different animals. $(G, H)$ DAPI staining of intestinal (arrowheads) and distal germ (arrows) cells. Contrast the large, brightly staining intestinal cells in $G$ to the smaller, fainter intestinal cells in $H$. A relative indication of intestinal-cell DNA content can be inferred from comparing the intensity of intestinal-cell staining to that of the germ cells in each panel. $(I, I)$ Proximal gonad and vulval region in adults. White arrowheads indicate the location of vulval eversions. Note the presence of multiple eversions in $J$. In addition, the animal in $J$ shows a hyperproliferation defect of the proximal germ line (pg), whereas the animal in $G$ contains wild-type embryos (em) in this region. The distal germ line (dg) appears normal in both $I$ and $J$. Size bars, $10 \mu \mathrm{m}$. tra DTCs, leading to a Shiva (Shv) phenotype, in which an abnormal bifurcation produces gonads with extra arms (Fig. 3C,D; Table 2). In general, only one bifurcation per gonad arm was observed, indicating that most DTCs were undergoing only a single extra division cycle. Consistent with this, the average number of DTCs detected using the DTC-expressing lag-2::GFP marker (Blelloch et al. 1999) was $1.67 \pm 0.68$ (range, $1-4 ; n=60$ ) per gonad arm, where only 5/60 animals contained $>2$ DTCs per arm. An analysis of DTC divisions in $f z r-1$; lag-2::GFP; lin-35(RNAi) animals indicated that most or all extra DTCs are generated through division of the DTC itself, and not from other cell types in the somatic gonad. This result was supported by the direct observation of lag-2::GFP-expressing DTCs in the process of division (Fig. 3E,F), and by experiments where DTCs were laser-ablated in L2-stage animals: 6/7 animals failed to produce additional DTCs based on gonad morphology and lag-2::GFP expression. This result contrasts with findings in cki-1(RNAi) animals where supernumerary DTCs were shown to originate from other cells within the somatic gonad (Hong et al. 1998). We cannot, however, rule out the possibility that a small percentage of DTCs in lin-35; fzr-1 animals may be derived from other sources.

Endoderm was also strongly affected in lin-35; $f z r-1$ double mutants. Wild-type adults normally contain $\sim 26$ gut cell nuclei that are polyploid $(32 \mathrm{~N})$ as a consequence of four endoreduplication cycles (DNA replication without intervening mitosis) during larval development (Fig. 3G; Hedgecock and White 1985). In lin-35; fzr-1 double mutants, gut cell ploidy was highly variable, ranging from $2 \mathrm{~N}$ to $16 \mathrm{~N}(n=20)$, with the majority of cells falling in the range of $4 \mathrm{~N}$ to $8 \mathrm{~N}$ (Fig. $3 \mathrm{H}$; data not shown). In

Table 2. fzr-1 genetic interactions with SynMuv genes

\begin{tabular}{|c|c|c|}
\hline & $\%$ Shv & $n$ \\
\hline $\operatorname{lin}-35(n 745)$ & 1 & 76 \\
\hline fzr-1(ku298) & 0 & 40 \\
\hline lin-35; fzr-1; kuEx119 & 2 & 97 \\
\hline lin-35; $f z r-1$ & 58 & 45 \\
\hline lin-35; fzr-1; kuEx119; lin-35(RNAi) ${ }^{\mathrm{a}}$ & 68 & 60 \\
\hline lin-35; fzr-1; kuEx119; lin-36(RNAi) & 69 & 36 \\
\hline lin-35; fzr-1; kuEx119; efl-1(RNAi) ${ }^{a}$ & 46 & 26 \\
\hline$f z r-1 ; \operatorname{lin}-53(n 833)$ & 0 & 25 \\
\hline lin-35; fzr-1; kuEx119; lin-53(RNAi) ${ }^{\mathrm{b}}$ & 4 & 28 \\
\hline lin-35; fzr-1; kuEx119; hda-1(RNAi) ${ }^{\mathrm{b}}$ & 0 & 25 \\
\hline lin-35; fzr-1; kuEx119; chd-4(RNAi) & 0 & 43 \\
\hline fzr-1; lin-15a(n767) & 0 & 22 \\
\hline lin-35; fzr-1; kuEx119; lin-15a(RNAi) & 7 & 81 \\
\hline lin-35; kuEx119; fzr-1(RNAi) & 2 & 61 \\
\hline lin-35; fzr-1(RNAi) & 37 & 49 \\
\hline
\end{tabular}

Adult hermaphrodites of the indicated genotypes were placed on RNAi feeding plates, and adult $\mathrm{F}_{1}$ progeny were scored for the presence of the Shiva (Shv) phenotype. kuEx119 is the extrachromosomal array containing the 1 in $-35^{+}$gene.

aSimilar percentages of Shv animals were also observed following RNAi treatment of $f z r-1$ single mutants.

${ }^{b}$ RNAi treatment led to a highly penetrant sterile phenotype among $F_{1}$ progeny. 
addition, we observed lin-35; fzr-1 DAPI-stained animals that contained up to four times the normal number of intestinal cell nuclei (Fig. $3 \mathrm{H})$. Consistent with this, fzr1; lin-35(RNAi) animals expressing the gut-cell-specific marker elt-2::GFP showed excess divisions (Table 1). The large number and reduced ploidy of mutant gut cells suggest that rather than executing endocycles during larval development, intestinal cells in the double mutants are undergoing cycles of DNA replication followed by mitosis. Interestingly, both lin-35 and $f z r-1$ single mutants showed a modest increase in the number of gut cell nuclei compared with wild type (Table 1).

Excess divisions were also observed in cells that make up the germ line. Severe effects were confined to proximal regions of the gonad, where rampant hyperproliferation led in some cases to a tumorous phenotype (Fig. 3I, J; Francis et al. 1995). The specific involvement of germ cells was evidenced by the lack of any detectable GFP expression in affected regions of the gonad in MH1621 animals in which lin-35 was inactivated with RNAi (data not shown). This failure of the tumorous cells to express GFP reflects the ability of germ-line cells to efficiently silence transgene expression, a characteristic not associated with the somatic gonad, in which we observed consistent GFP expression. Distal gonad regions were less affected and showed normal-appearing DAPIstained mitotic, transition, and pachytene zones (data not shown). These results suggest that germ cells in lin35; fzr-1 mutants abnormally reenter mitosis after failing to progress past meiotic prophase. These defects could reflect a direct requirement for lin-35 in germ cells, or may be caused by nonautonomous effects, such as signaling via the lag-2/glp-1 pathway from the somatic gonad.

fzr-1 shows synthetic phenotypes with a subset of class B SynMuv genes

As described in the introduction, a subset of class B SynMuv genes (including lin-35) have been linked to transcriptional regulation. However, it is currently unclear to what extent these functionally related genes are interchangeable with respect to their genetic interactions. To address this issue, we examined interactions between $f z r-1$ and six SynMuv genes. The class B genes efl-1, lin53, hda-1, and chd-4 encode homologs of E2F (Ceol and Horvitz 2001), RbAp48, histone deacetylase (Lu and Horvitz 1998), and the NuRD complex component, Mi-2, respectively (Solari and Ahringer 2000; von Zelewsky et al. 2000). lin-15a and lin-36 (class A and B genes, respectively) encode novel proteins (Clark et al. 1994; Huang et al. 1994; Thomas and Horvitz 1999).

Similar to the effects observed for lin-35(RNAi) (Fig. $2 \mathrm{G}, \mathrm{H}$; Tables 1,2$)$, RNAi inactivation of either lin-36 or efl-1 in strain MH1621 led to the appearance of kuEx $119^{+}$animals that were phenotypically indistinguishable from lin-35; fzr-1 double mutants (Table 2; data not shown). In contrast, RNAi inactivation of lin53, hda-1, chd-4, and lin-15a failed to induce hyperproliferation in kuEx119+ animals (Table 2; data not shown).
In addition, we constructed double mutants between fzr-1 and lin-53 and lin-15a. In both cases, double-mutant animals were indistinguishable from wild type with respect to viability and cellular proliferation properties (Table 2; data not shown). We conclude that fzr-1 shows interactions with a subset of class $B$ genes that may function more specifically to control cell cycle regulation.

\section{fzr-1 encodes a C. elegans homolog of Cdh1/Hct1/FZR}

Using several novel approaches to overcome a number of technical challenges (see Materials and Methods), we mapped ku298 to a small region on LGII between let-23 and unc-4. We attained rescue of the lin-35; fzr-1 doublemutant phenotype using microinjection with a single cosmid from the region ZK1307. Partial but clear rescue was subsequently obtained with a subclone of ZK1307 that contained only a single predicted ORF, ZK1307.6 (see Materials and Methods). A search of the database indicated that ZK1307.6 encodes the C. elegans homolog of Cdh1/Hct1/FZR.

To further support the claim that ku298 represents an allele of $f_{z r}-1$, we sequenced $f_{z r}-1$ genomic DNA from ku298 mutant animals. We detected a single missense mutation within the predicted coding region of $f_{z r}-1$. The mutation $(\mathrm{G} \rightarrow \mathrm{A})$ substitutes a highly conserved cysteine with tyrosine in the fourth WD-repeat domain (Fig. 4A). As an additional test, strain MH1461 was grown on $f z r-1(R N A i)$ feeding plates. Although kuEx $119^{+}$animals appeared largely unaffected by this treatment, the majority of kuEx119- animals showed hyperproliferation defects similar to those of lin-35; fzr-1 double mutants (Fig. 5A; Table 2; data not shown).

Using a cDNA clone (gift of Y. Kohara, National Institute of Genetics, Mishima, Japan), we confirmed the predicted gene structure of $f z r-1$ and identified an SL1 transplice leader sequence (Krause and Hirsh 1987) at the 5 ' end of the clone, indicating that it is full length. The highly conserved C-terminal domain of FZR-1 is $68 \%$ identical to both Drosophila FZR and human CDH1 (Fig. 4A). The sizable N-terminal domain of FZR-1 contains scattered regions of homology to the fly and human gene products as well as novel sequences (Fig. 4A). A phylogenetic analysis indicates that FZR-1 is more similar to members of the Cdh1/Hct1/FZR family than to the closely related but functionally distinct gene family composed of Cdc20/FZY (Fig. 4B).

\section{FZR-1 function is required for fertility}

The nature of the genetic lesion suggested that ku298 might not represent a complete LOF allele of $f z r-1$. Because dsRNA injection techniques may produce RNAi phenotypes that more closely mimic that of the molecular null than do those produced by feeding methods (M. Han, unpubl.), we inactivated FZR-1 function in wild-type animals using RNAi injection methods. Injection led to a severe reduction in the fertility of the de- 
rived $F_{1}$ progeny: $47 \%$ of $F_{1}$ animals were completely sterile, whereas an additional $39 \%$ had brood sizes of $<10$ $(n=210)$. An analysis of affected animals revealed pleiotropic defects within the germ-line and somatic gonad. These included a failure of gonad arms to elongate, missing or abnormal oocytes and sperm, and morphogenetic defects of the uterus and vulva (Fig. 5B; data not shown). From these results, we conclude that FZR-1 function is required for viability and that ku298 encodes a hypomorphic allele of $f z r-1$.

We also examined the effect of inactivating $f z r-1$ using RNAi injection methods in a lin-35 mutant background. Interestingly, this led to embryonic lethality in $88 \%$ of $\mathrm{F}_{1}$ progeny, with the remaining $12 \%$ arresting during early larval stages $(n=269)$. Nonviable embryos showed a variety of phenotypes ranging from premorphogenetic to late-stage arrest (Fig. 5C,D). In the majority of cases, arrested embryos showed clear signs of tissue-specific differentiation but failed to complete morphogenesis and contained vacuoles and necrotic regions (Fig. 5C,D). In addition, a significant percentage of embryos ( 30\%) were misshapen or of abnormal size (Fig. 5D). We conclude that, in addition to controlling cell proliferation during postembryonic development, lin-35 and $f z r-1$ play essential, albeit redundant, roles during embryonic development.

Consistent with these findings, an $f z r-1:: G F P$ transcriptional reporter showed robust expression in most or all cells during embryonic development in wild-type animals (Fig. 5E,F). Expression was also observed at lower levels in many cell types during larval development, including those showing proliferation defects in lin-35; $f z r-1$ double mutants (data not shown).

lin-35 shows a synthetic interaction with the putative SCF regulator lin-23

lin-23 encodes a WD-repeat protein with homology to regulators of the SCF proteosome complex (for review, see Deshaies 1999; Kipreos et al. 2000). Loss of LIN-23 function leads to a widespread hyperproliferation phenotype in mutant animals, presumably resulting from a failure to degrade $G_{1}$ cyclins. We had previously identified an allele of lin-23 in an earlier screen for lin-35 synthetic mutants (D.S.F. and M.H., unpubl.). lin-23(ku294) contains a mutation in the conserved $5^{\prime}$ splice site (GT $\rightarrow \mathrm{AT}$ ) between exons 4 and 5 of lin-23, resulting in a peptide that is predicted to terminate following amino acid 245 of the 665 -amino-acid peptide. Based on the size of the truncation and the predicted instability of the resulting mRNA, ku294 is likely to represent a strong or complete LOF allele of lin-23.

In contrast to lin-23 and lin-35 single mutants, lin-35; lin-23(ku294) double mutants generally fail to progress past the L3 larval stage and are severely growth retarded relative to the single mutants (Fig. 6A; data not shown). Because of the extremely poor viability of lin-35; lin-23 double mutants, and the fact that lin-23 single mutants themselves show a strong hyperproliferation phenotype on their own, it was not possible for us to discern whether the absence of LIN-35 function exacerbated the lin-23 hyperproliferation defect in the double mutants.

As an alternative approach, we reduced lin-23 function in MH1461 animals using RNAi feeding methods and looked for differences in the sensitivity of lin-35; kuEx119+ and lin-35; kuEx119- sibling progeny. Interestingly, whereas $82 \%(n=177)$ of $k u E x 119^{-}$animals exposed to lin-23(RNAi) showed the characteristic lin-23 hyperproliferation phenotype, only $43 \%(n=236)$ of sibling kuEx $119^{+}$animals were similarly affected (Fig. $6 \mathrm{~B}, \mathrm{C})$. Thus the presence of functional LIN-35 significantly decreased sensitivity to diminished LIN-23 activity. This effect was not due to a generic hypersensitivity of lin-35 mutant animals to RNAi as both $\mathrm{kuEx} 119^{+}$ and $\mathrm{kuEx} 119^{-}$animals were equally sensitive to unc22(RNAi) feeding (data not shown).

\section{LIN-35 and FZR-1 likely coregulate $G_{1}$ cyclin levels}

Based on work from other systems (see Discussion), loss of LIN-35 function would be predicted to disrupt the activity of $\mathrm{Rb} / \mathrm{E} 2 \mathrm{~F}$ transcriptional inhibitory complexes, leading to elevated levels of cyclin E and cyclin A mRNA. In turn, loss of either FZR-1 or LIN-23 would result in decreased APC or SCF functions, leading to an increase in the stability of cyclin A and cyclin E proteins, respectively. A simple model would then predict that in the lin-35; fzr-1 double mutant, the combination of both increased cyclin transcription and stability leads to cyclin protein levels that are sufficient to override the normal $G_{1}$ arrest.

To test this model, we engineered strains containing temperature-inducible cyclin A or cyclin E constructs expressed from extrachromosomal arrays. As shown in Table 3, in an otherwise wild-type background, either cyclin A or cyclin E overexpression can lead to the production of extra distal tip cells in a small percentage of animals. Notably, this effect is strongly enhanced in an $f z r-1$ mutant background. The synthetic interaction observed between cyclin overexpression and $f z r-1$ mutants is consistent with the model that LIN-35 and FZR-1 cooperate to control the levels of $\mathrm{G}_{1}$ cyclins through transcriptional repression and protein destabilization, respectively.

\section{Discussion}

\section{lin-35 and the control of cell proliferation}

We report here the cloning and characterization of $f z r-1$, a gene that cooperates with lin-35 to control cell proliferation. A relatively small number of genes have been described that cause widespread hyperproliferation in $C$. elegans. These include the putative SCF components cul-1 and lin-23 (Kipreos et al. 1996, 2000), the CIP/KIP family member cki-1 (Hong et al. 1998), and the CBP/ p300 homolog cbp-1 (Shi and Mello 1998). In the cases examined thus far, a hyperproliferation phenotype is observed following inactivation of a single gene product. 
Fay et al.

\section{A}

\begin{tabular}{ll}
$\begin{array}{l}f z r-1 \text { C. elegans } \\
f z r D \text {. melanogaster }\end{array}$ & 1 \\
\hline
\end{tabular} Cdhl human

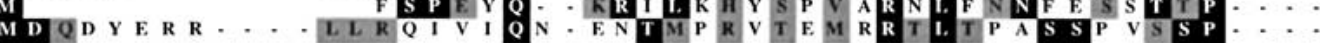

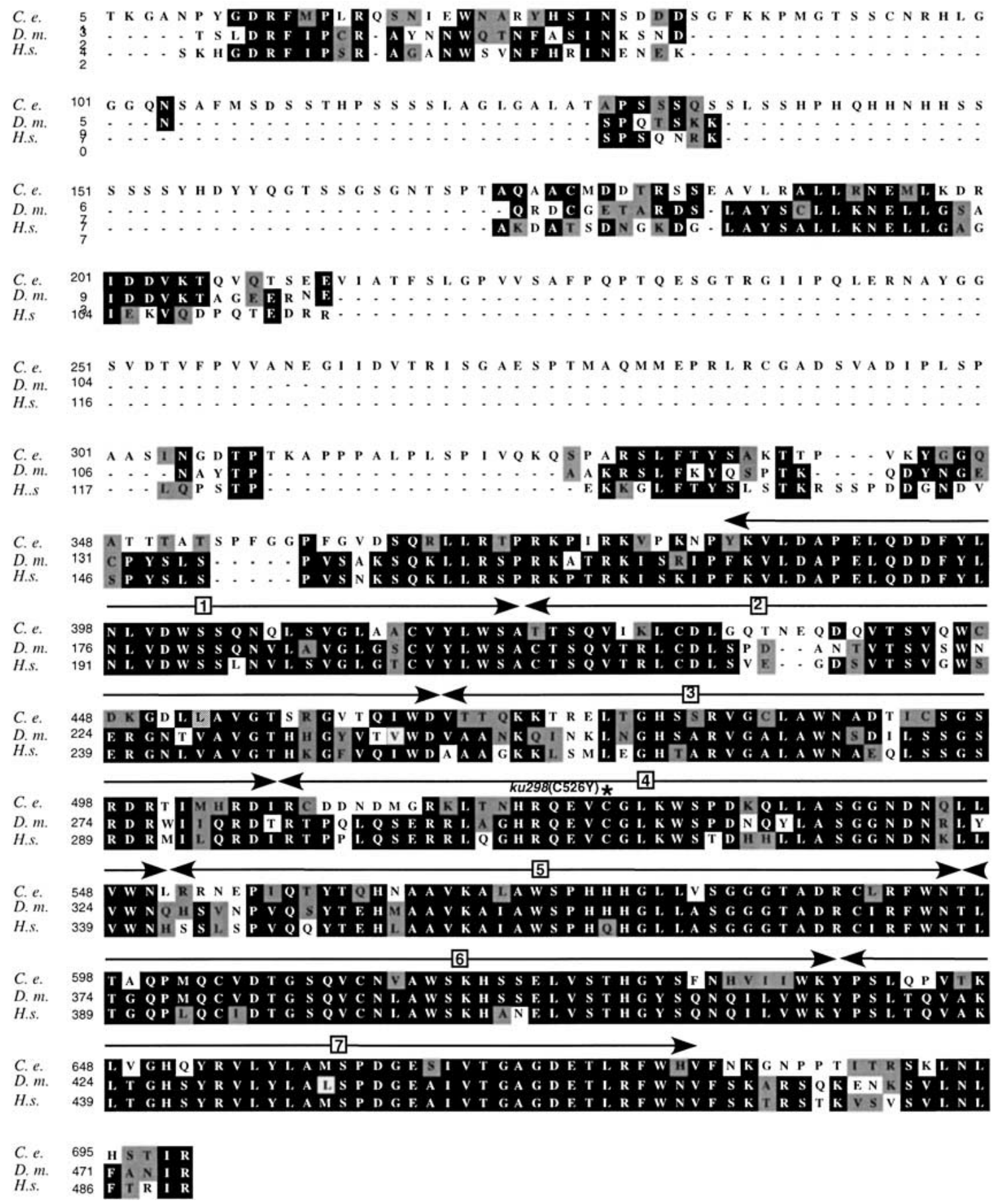

(Figure 4 continued on facing page)

We have shown that loss of cell proliferation control can also result from a synthetic genetic interaction. Although single mutants of lin-35 and fzr-1 showed only subtle or low-penetrance phenotypes, Iin-35; fzr-1 double mutants showed extensive tissue hyperproliferation affecting a wide range of cell types (Figs. 3-5; Tables 1, 2). Thus, uncontrolled proliferation in C. elegans can in essence follow the same genetic pattern as multistep car- 


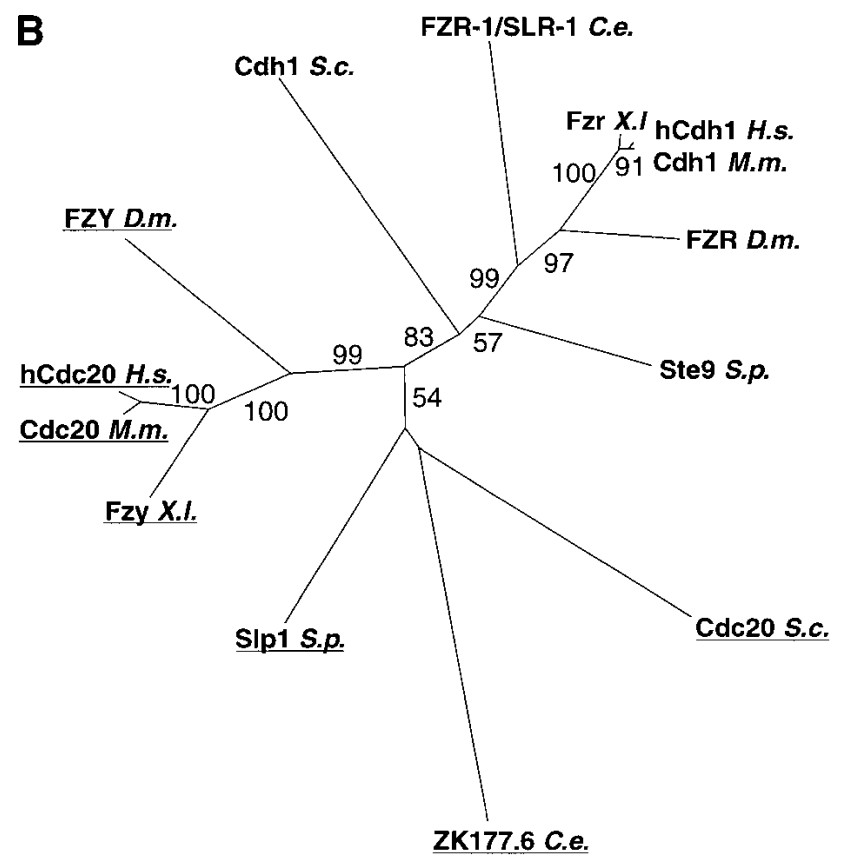

Figure 4. (A) Peptide alignment of C. elegans FZR-1, Drosophila melanogaster Fzr, and Homo sapiens CDH1. Black boxes signify identity; gray, similarity. The boundaries of the seven WD-repeat regions are indicated by arrows and corresponding numbers. The location of the ku298 lesion is indicated by an asterisk. $(B)$ Unrooted phylogenetic tree diagram showing relationships between FZR (non-underlined) and FZY (underlined) homologs from C. elegans, D. melanogaster, Xenopus laevis, Mus musculus, H. sapiens, Saccharomyces cerevisiae, and Schizosaccharomyces pombe. Numbers indicate bootstrap values.

cinogenesis in mammals. Namely, proliferation control is abolished through the sequential loss of genes that function to restrain cell cycle progression.

Given our findings, and the large body of evidence implicating $R b$ in human cancers (for review, see Nevins 2001), it seems reasonable to suggest that our technical approach may facilitate the study of multistep carcinogenesis using C. elegans. Along these lines, it will be interesting to determine whether the human homolog of $f z r-1, \mathrm{hCDH} 1$, can function as a tumor-suppressor gene, and if so, whether it does so in cooperation with $\mathrm{Rb}$.

Inactivation of $f z r-1$ function using RNAi injection led to sterility and aberrancies in germ cell proliferation (Fig. $5 \mathrm{~A}, \mathrm{~B})$. Although we have not determined the specific cause of this phenotype, previous studies would implicate defects in either the execution of $\mathrm{G}_{1}$ arrest (Irniger and Nasymth 1997; Sigrist and Lehner 1997; Zachariae et al. 1998) or in late-stage mitotic events such as cytokinesis (Schwab et al. 1997; Zur and Brandeis 2001). We also observed embryonic lethality when $f z r-1$ was inactivated using RNAi injection in a lin-35 mutant background (Fig. 5C,D). The cause for this lethality is presently unknown. These embryos do not show obvious hallmarks associated with either excess cellular proliferation or grossly elevated levels of apoptosis (data not shown). Although additional work will be necessary to determine the nature of this embryonic requirement, a role during embryonic development is consistent with the expression patterns observed for both $f_{z r}-1$ (Fig. 5E,F) and lin-35 (Lu and Horvitz 1998). The lack of an apparent hyperproliferation phenotype in embryos likely reflects significant differences in the means by which embryonic and postembryonic cell cycles are regulated. For example, cyclin $\mathrm{D}$, an upstream regulator of $\mathrm{Rb}$, has been shown to be required exclusively for the execution of postembryonic division cycles in C. elegans (Park and Krause 1999; Boxem and van den Heuvel 2001).

\section{Differential properties of SynMuv genes}

Work carried out over the past several years has produced an explosion in the number of identified SynMuv genes (see Fay and Han 2000). Although certain functional classifications, such as transcriptional repressors, may accurately describe some of the SynMuv genes, others clearly defy straightforward categorization. This fact alone suggests that SynMuv genes most likely do not all act through the same mechanisms or pathways.

We have found that both lin-36 and efl-1(RNAi) can phenocopy the effect of lin-35 LOF in an fzr-1 mutant background (Table 2). However other class B genes, including lin-53, hda-1, and chd-4, did not show genetic interactions with $f z r-1$, nor did the class A gene lin-15a (Table 2). These experiments are complicated by the fact that lin-53, hda-1, and chd-4 encode for essential genes, and RNAi leads to a highly penetrant sterile or lethal phenotype within several generations. Nevertheless, we saw no evidence for hyperproliferation in either the affected or unaffected classes of RNAi-treated animals. This suggests that neither a weak nor a severe reduction in the function of these genes is capable of producing a synthetic hyperproliferation phenotype with $f z r-1$. In addition, we detected no evidence for an interaction in lin53(n833); fzr-1 double-mutant animals. Although $n 833$ results in only a partial loss of LIN-53 function (Lu and Horvitz 1998), this allele does lead to a highly penetrant Muv phenotype in conjunction with class A SynMuv mutations (Ferguson and Horvitz 1989).

These data indicate a functional distinction between those class B genes that may play relatively direct roles in the regulation of the cell-cycle machinery (Iin-35, lin36 , and efl-1) and others that may function more generally in the regulation of chromatin modification and transcriptional control (lin-53, hda-1, and chd-4). Moreover, our data correlate well with recent studies on vulval-cell fate specification, in which clear functional differences were observed between similar subsets of class B genes (Chen and Han 2001).

\section{lin-35, fzr-1, lin-23, and cyclin regulation}

$\mathrm{Rb}$ and its family members p107 and p130 have been shown in multiple systems to modulate transcription through direct interactions with a variety of transcrip- 
Fay et al.

Figure 5. $f z r-1(R N A i)$ and $f z r-1$ expression. (A) Example of the Shv phenotype observed in lin-35 animals grown on $f z r$ 1(RNAi) feeding plates. $(B)$ Representative gonad from progeny of $\mathrm{N} 2$ animals injected with fzr-1 dsRNA (RNAi; $1.5 \mu \mathrm{g}$ / $\mu L)$. Note the small malformed gonad arm extension (arrow) protruding from the proximal gonad region (pg). $(C, D)$ Terminal embryos derived from lin-35 animals injected with $f_{z r}-1$ dsRNA. Arrows indicate the locations of vacuoles. Note the variability in embryonic $(C, D)$ arrest point and $(D)$ size. $(E, F)$ Corresponding DIC and GFP fluorescence images of an $f z r-1:: G F P$ reporter construct expression in wild-type embryos. Size bars, $10 \mu \mathrm{m}$.
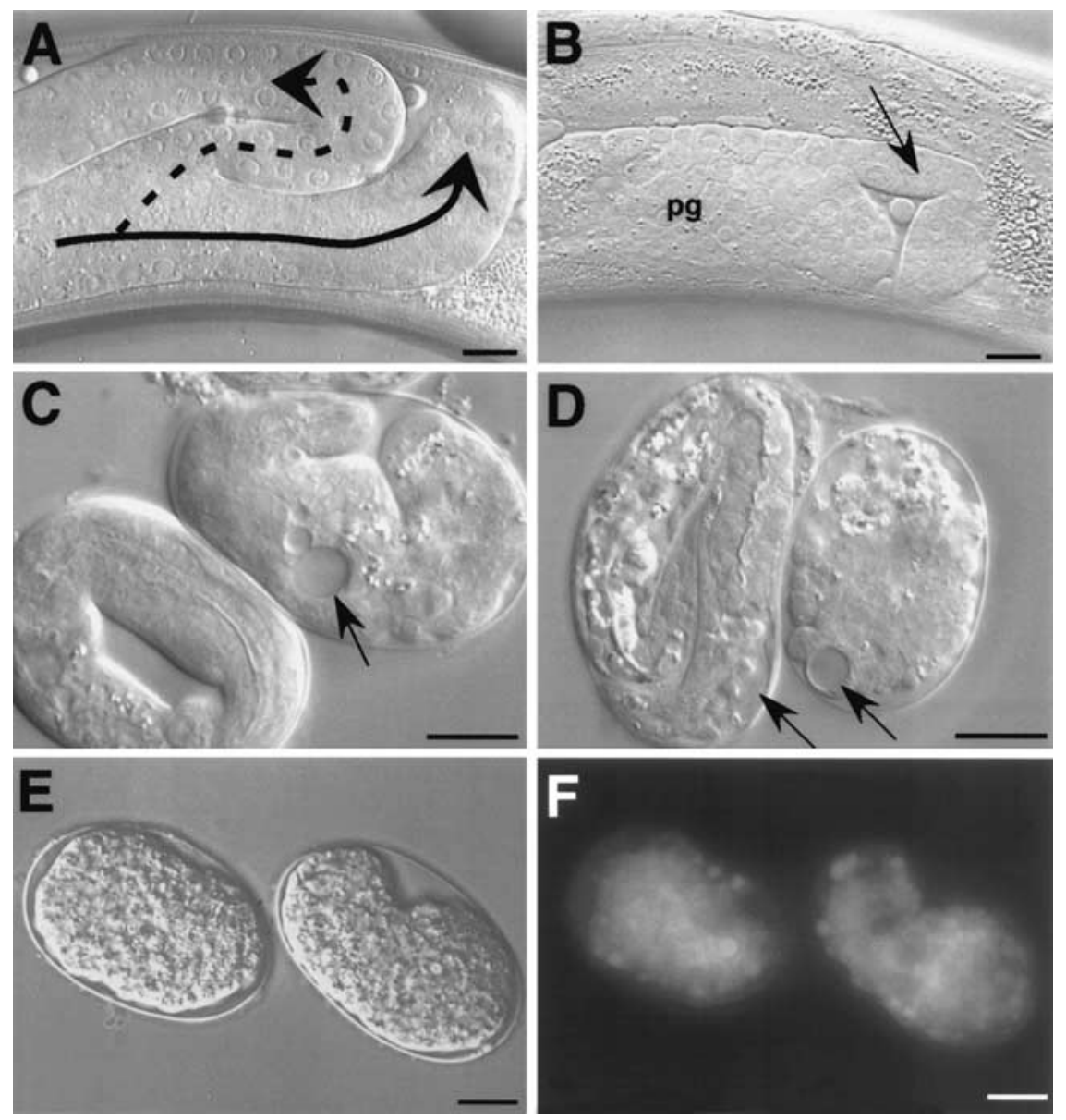

tional regulators (for review, see Kaelin 1999; Zheng and Lee 2001). The majority of work indicates that $\mathrm{Rb}$ serves primarily as a transcriptional repressor, acting through a number of mechanisms including the recruitment of chromatin-modifying enzymes (Dunaief et al. 1994; Brehm et al. 1998; Luo et al. 1998; Magnaghi-Jaulin et al. 1998; Zhang et al. 2000; Nielsen et al. 2001) and the steric interference of transactivation domains (Flemington et al. 1993; Helin et al. 1993; Weintraub et al. 1995). Acting as transcriptional corepressors with E2F (for review, see Dyson 1998; Harbour and Dean 2000; Classon and Dyson 2001), Rb and its family members regulate the expression of many key genes required for entry and progression through S-phase (Dou et al. 1994) including cyclin E (DeGregori et al. 1995; Duronio and O'Farrell 1995; Ohtani et al. 1995) and cyclin A (Degregori et al. 1995; Schulze et al. 1995). Consistent with these reports, we have observed a significant increase in the levels of ribonucleotide reductase mRNA, an E2F-regulated gene, in lin-35 mutant animals (D.S. Fay and M. Han, unpubl.).

In contrast, Cdh1/Hct1/FZR and its close relative Cdc20/FZY function as regulatory subunits for the anaphase-promoting complex (APC) cyclosome (Dawson et al. 1995; Sigrist et al. 1995; Schwab et al. 1997; Visintin et al. 1997; for review, see Morgan 1999; Zachariae and Nasmyth 1999). This multisubunit peptide complex is thought to function as a ubiquitin ligase, and targets the degradation of several key factors during mitosis, including yeast Pds1 (Cohen-Fix et al. 1996), human securin/ PTTG (Zur and Brandeis 2001), and cyclins A and B in higher eukaryotes (Dawson et al. 1995; Irniger et al. 1995; King et al. 1995; Sigrist et al. 1995; Sudakin et al. 1995). APC function requires activation first by Cdc20/ FZY during metaphase/anaphase, and then by Cdh1/ Hct/FZR during late mitosis and $G_{1}$. It is believed that Cdh1 and Cdc20 differentially target distinct but overlapping subsets of proteins for degradation by the APC cyclosome (see above references; for review, see Morgan 1999; Zachariae and Nasmyth 1999).

In Drosophila, loss of $f z r$ function leads to reentry into the cell cycle following embryonic cycle 16, thereby bypassing the normal $\mathrm{G}_{1}$ arrest (Sigrist and Lehner 1997). This ectopic division cycle is correlated with excess levels of cyclin A (Sigrist and Lehner 1997), which when overexpressed during $G_{1}$ can lead to ectopic entry into S-phase (Sprenger et al. 1997). Interestingly, mutations in the Drosophila Rb homolog rbf ( $\mathrm{Du}$ and Dyson 1999), as well as in the CDK inhibitor decapo (de Nooij et al. 1996), show cell cycle defects similar to those of $f z r$ mutants, suggesting complementary roles in $\mathrm{G}_{1} / \mathrm{S}$-phase regulation. However, we note that conclusions regarding $f z r$ functions were inferred from the analysis of a large 

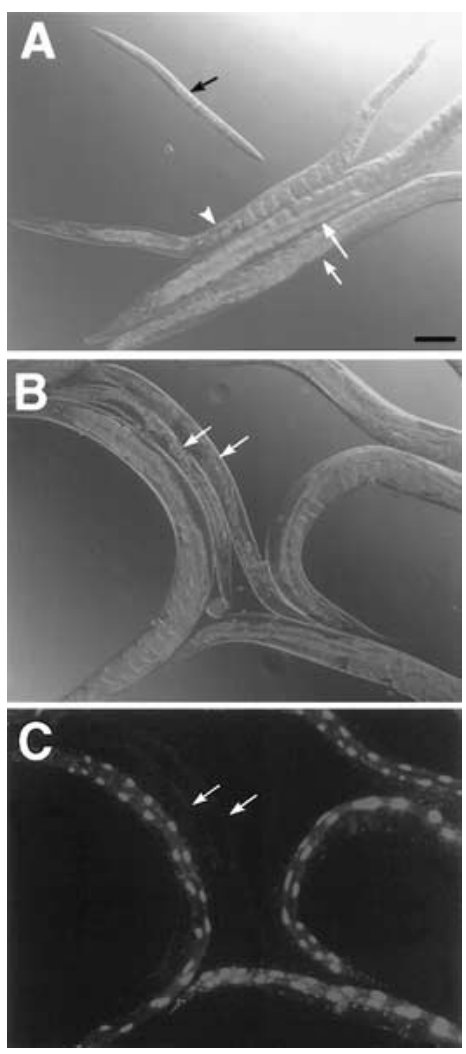

Figure 6. lin-35; lin-23 synthetic interactions. (A) lin-35 single mutant (white arrows), lin-23(ku294) single mutant (white arrowhead), and lin-35; lin-23 double mutants (black arrow). The lin-35; lin-23 animal shown is $>96 \mathrm{~h}$ posthatching and shows the typical midlarval-stage terminal arrest phenotype. $(B, C)$ Corresponding DIC and GFP fluorescence images of representative adult MH1461 animals propagated on lin-23(RNAi) feeding plates. White arrows indicate the positions of Ex119- animals. Note the preferential effect of lin-23(RNAi) on the kuEx119- animals. Size bar, $100 \mu \mathrm{m}$.

deletion that removed several genes in addition to $f z r$ (Sigrist and Lehner 1997). Therefore, fzr-1(ku298) is the first reported mutation in metazoans that specifically reduces $\mathrm{CDH} 1 / \mathrm{HCT} 1 /$ fzr activity.

Our analysis of DTC hyperproduction in strains that overexpress either cyclin A or cyclin E mRNA (Table 3) supports the model that lin-35 and fzr-1 are likely to coregulate cyclin levels during $\mathrm{G}_{1}$. In addition, our finding that the E2F homolog efl-1 synergizes with fzr-1 (Table 2) adds further credence to this model. The ability of both cyclin A and cyclin E to induce extra DTCs in fzr-1 mutants could indicate that these cyclins may be functionally interchangeable and that sufficient levels of either cyclin A or cyclin E, or possibly both in combination, can work to override $G_{1}$ arrest.

\section{Multiple mutations show synthetic lethality} with lin-35

By screening $~ 3500$ haploid genomes, we uncovered seven Slr mutations that show synthetic lethality or in- viability with mutations in lin-35. The phenotypes observed in the double mutants range from embryonic and early larval arrest to adult sterility and size defects (D.S. Fay and M. Han, unpubl.). About half of the Slr mutations that we have identified show only little or no phenotype as single mutants, whereas others show moderate effects on brood size and/or growth rates. In addition, we note that the penetrance and severity of the double-mutant phenotypes appear in several cases to be substantially reduced by the presence of low levels of maternal lin-35 expressed from the array, consistent with previous reports that maternal lin-35 can also suppress expression of the Muv phenotype (Ferguson and Horvitz 1989).

Other than fzr-1 and lin-23, we have not yet identified any Slr mutations that produce an obvious synthetic hyperproliferation phenotype with lin-35. However, as inferred from our comparison of lin-35(n745); fzr-1(ku298) and lin-35(n745); fzr-1(RNAi) phenotypes, this may depend to a significant extent on the specific nature of the allele isolated. Therefore, whether or not additional Slr genes will turn out to function in cell cycle control mechanisms or will cooperate with lin-35 in activities not directly related to the cell cycle remains to be seen.

\section{A screen of general utility}

We believe the means used to uncover the genetic interaction between lin-35 and fzr-1 will be of general use for those wishing to assign functions to genes lacking known biological roles or to identify novel functions for genes with previously characterized activities. In addi-

Table 3. Heat shock of cyclin $A$ and cyclin $E$

\begin{tabular}{lrr}
\hline & $\%$ Shv & $n$ \\
\hline Cyclin A overexpression & & \\
$\quad$ zr-1; $(f d E x 1)$ & 16 & 154 \\
$f z r-1$ & 0 & 91 \\
N2; $(f d E x 1)$ & 5 & 78 \\
N2 & 0 & 70 \\
$f z r-1 ;(f d E x 2)$ & 32 & 122 \\
$f z r-1$ & 1 & 69 \\
N2; fdEx2 & 5 & 86 \\
N2 & 0 & 63 \\
Cyclin E overexpression & & \\
$f z r-1 ;(f d E x 6)$ & 18 & 122 \\
$f z r-1$ & 0 & 115 \\
N2; $(f d E x 6)$ & 3 & 64 \\
N2 & 0 & 42 \\
$f z r-1 ;(f d E x 7)$ & 5 & 124 \\
$f z r-1$ & 1 & 127 \\
N2; $(f d E x 7)$ & 1 & 62 \\
N2 & 0 & 50
\end{tabular}

Strains harboring cyclin A $(f d E x 1, f d E x 2)$ or cyclin E (fdEx6, $f d E x 7)$ heat-shock expression constructs contained on extrachromosomal arrays were generated as described in Materials and Methods. Mixed-stage L1/L2 larval animals were heatshocked at $33^{\circ} \mathrm{C}$ for $4 \mathrm{~h}$ and allowed to recover at $20^{\circ} \mathrm{C}$ for $\sim 52$ h. Sibling $E x^{+} / E x^{-}$young adult animals were then analyzed for the Shiva phenotype. Percentages indicate the averages obtained from two separate experiments. 
tion, this genetic approach serves to identify functional copartners through the isolation and cloning of the affected second-site mutations. Importantly, this method in no way depends on prior knowledge of the synthetic double-mutant phenotype, thereby permitting a nonbiased search for genetic modifiers of any gene of interest.

Given the inevitable saturation of the genome for mutations that cause easily detectable phenotypes, the ability to identify synthetic mutations will become increasingly important. Large-scale analyses carried out in yeast and $C$. elegans suggest that a large percentage of genes in higher organisms may fail to show easily discernable phenotypes when mutated (Smith et al. 1996; Winzeler et al. 1999; Fraser et al. 2000). It is likely that the vast majority of these no-phenotype genes may nevertheless confer a weak selective advantage to the organism, thus accounting for their presence in the genome (Nowak et al. 1997; for review, see Tautz 2000). At the same time, it can be argued that many of these genes fail to show mutational effects owing to genetic redundancy. Importantly, these two explanations are in no way mutually exclusive. By devising methods to experimentally address this latter issue, we may hope to assign biological roles to many genes that would normally not be amenable to straightforward functional analyses.

\section{Materials and methods}

\section{Culture methods}

Maintenance, culturing, and genetic manipulations of C. elegans strains were carried out according to standard procedures (Sulston and Hodgkin 1988) and conducted at $20^{\circ} \mathrm{C}$.

\section{Strains used/generated}

The strains used or generated were as follows: MT1624 [lin35(n745)I; lin-8(n111)II], MH1461 [lin-35(n745)I; kuEx119], MH1621 [lin-35(n745)I; fzr-1(ku298)II; kuEx119], MH1829 [fzr1(ku298), unc-4(e120)II], MH1832 [dpy-10(e128), fzr-1(ku298), unc-4(e120)II], CB4856, MH1791 [fzr-1(ku298)II], MH1640 [lin-35(n745)I; dpy-10(e128), unc-4(e120)II], MH1630 [lin35(n745)I; bli-2(e768), rol-6(e187)II], MH1758 [lin-35(n745)I; let-23(sy15), unc-4(e120)/dpy-10(e128), unc-4(e120)II], JK2533 [qC1(lag-2::GFP qIs26)/eT1 (Blelloch et al. 1999)], GR1314 [mgIs21 (lin-11::GFP (Hobert et al. 1998)], JR632 [seam cell::GFP (Terns et al. 1997)], oxIn12 [unc-47::GFP (McIntire et al. 1997), and JM63 [elt-2::GFP (Fukushige et al. 1998)].

\section{DNA constructs}

RNAi Target sequences for insertion into pPD129.36 (gift of A. Fire) were generated by PCR amplification from appropriate cDNA or genomic clones. cDNA clones of lin-35, efl-1, lin-36, $h d a-1$, and lin-53 were obtained from Y. Kohara, and a cDNA clone of lin-15a was obtained from P. Sternberg (California Institute of Technology, Pasadena). Inserts into pPD129.36 were of the following sizes: lin-35, 2.7-kb cDNA spanning exons 3-10. fzr-1, 750-bp genomic spanning exons 1-2. lin-23, 1.1-kb genomic spanning exons $2-5$. lin-15a, 1.1-kb cDNA spanning exons 2-6. chd-4, 1.4-kb genomic spanning exons 1-2. efl-1, 370-bp cDNA spanning exons 1-3. lin-36, 1.0-kb cDNA span- ning exons 3-5. hda-1, 1.4-kb cDNA spanning exons 1-4. lin-53, $1.3-\mathrm{kb}$ insert spanning exons $1-3$.

fzr-1 subclones pDF63 contains a 7960-bp XbaI/SalI fragment from cosmid ZK1307 subcloned into pBluescript. pDF65 contains a 3250-bp PCR-amplified fragment from the $5^{\prime}$ UTR of $f z r-1$ cloned into plasmid pPD95.69 (gift of A. Fire). The construct creates an in-frame fusion of GFP containing an NLS to the first 6 amino acids of $f z r-1$.

Cyclin heat-shock constructs Full-length cyclin A cDNA from clone yk610 (from Y. Kohara) was amplified and inserted into pPD49.78 and pPD49.83 heat-shock vectors (from A. Fire). Cyclin E-heat shock vectors were a gift of M. Krause (NIH, Bethesda, MD).

\section{Screen methodology}

lin-35(n745); lin-8(n111) (MT1624) animals were injected with a mixture of cosmid C32F10 (100 $\mu \mathrm{g} / \mu \mathrm{L}$; Gu et al. 1998) and sur-5::GFP $(100 \mu \mathrm{g} / \mu \mathrm{L})$ to generate multiple independent strains carrying stable extrachromosomal arrays. Array kuEx119 suppressed expression of the Muv phenotype in $>90 \%$ of 1 in- 35 ; lin- 8 double mutants and showed an $\sim 75 \%$ transmission frequency. After crossing out the lin- 8 mutation to generate strain MH1461, animals were mutagenized with ethylmethanesulfonate (EMS) using standard procedures (Sulston and Hodgkin 1988). An $\mathrm{F}_{2}$ clonal screen was carried out in which four $\mathrm{F}_{2}$ animals were cloned per $\mathrm{F}_{1}$ (for additional details, see Results), resulting in an expected coverage of $\sim 1.35$ haploid genomes per $\mathrm{F}_{1}$ animal. Based on this estimate, we have screened $\sim 3500$ haploid genomes, or $\sim 2500 \mathrm{~F}_{1}\left(10,000 \mathrm{~F}_{2}\right)$ clones, to obtain seven unlinked mutations.

\section{Mapping}

Positional mapping of $f z r-1$ presented a number of technical challenges: (1) both fzr-1(ku298) and lin-35(n745) single mutants lack discernable plate phenotypes; (2) double-mutant animals are nonviable and difficult to distinguish under a dissecting scope; and (3) the severity of the double-mutant phenotype can be at least partially suppressed by maternal products. These difficulties were circumvented by the procedures described below.

Two- and three-point mapping Triple-mutant mapping strains carrying lin-35(n745) and two linked visible marker mutations (e.g., dpy and unc) were generated for each chromosome. Homozygous lin-35 males were mated to mapping strains, and male cross progeny were in turn mated to strain MH1621 (fzr-1; lin-35; kuEx119). kuEx119+ cross progeny that segregated the marker mutations were then identified and used for either twoor three-point mapping.

For two-point mapping, 100 kuEx119+ wild-type sibling animals were cloned to single plates and, based on the segregated progeny, were scored as either homozygous or nonhomozygous for ku298 and plus or minus for the marker mutations. ku298 showed strong linkage to markers on LGII: 31/31 ku298 homozygous animals failed to segregate $d p y-10$, unc- 4 markers, and 25/26 ku298 homozygous animals failed to segregate bli-2, rol-6 markers.

For three-point mapping, kuEx $119^{+}$sibling recombinants (e.g., Dpy-non-Unc) were identified and allowed to self-fertilize. Then $10-15 \mathrm{kuEx} 119^{+}$self-progeny of the recombinant phenotype were cloned, allowed to self-fertilize, and then scored both for the presence of homozygous ku298 and of the recombinant chromosomes. 
We obtained the following data for three-point mapping of ku298: 3/19 unc-4-non- $d p y-10$ recombinants acquired ku298; 28/29 bli2-non-rol-6 recombinants acquired ku298; 0/5 rol-6-non-bli-2 recombinants acquired ku298; 9/38 unc-4-non-let-23 recombinants acquired ku298.

SNP mapping We constructed the triple mutant strain MH1832 (dpy-10, ku298, unc-4) and verified the strain's ability to phenocopy the lin-35; ku298 double-mutant phenotype using lin-35(RNAi) feeding methods (Fraser et al. 2000). MH1832 hermaphrodites were then mated to CB4856 homozygous males, and hermaphrodite cross progeny were cloned and allowed to self-fertilize. Recombinant animals (e.g., Unc-non-Dpy) were identified, and homozygous recombinant animals were isolated in the next generation and tested for the presence of ku298 using lin-35(RNAi). Predicted SNPs in the region were identified using the C. elegans SNP database and were further verified by experimentation.

We obtained the following mapping data for SNP vr77f08.s1@477 on cosmid T15H9: For Unc-non-Dpy recombinants that retained ku298, 54/54 were N2 for the SNP; 7/7 Unc-non-Dpy recombinants that lost ku298 were CB4856 for the SNP; 5/5 Dpy-non-Unc recombinants that retained ku298 were N2 for the SNP. For SNP vr89b04.s1@165 on cosmid T10B9 the following data were obtained: 2/7 Unc-non-Dpy recombinants that lost ku298 were CB4856 for the SNP; 3/5 Dpynon-Unc recombinants that retained ku298 were CB4856 for the SNP.

\section{Other methods}

Cosmid rescue Rescue was obtained by coinjection of either ZK1307 or pDF63 with pRF4 into MH1621 using standard procedures (Mello and Fire 1995). Rescued animals were fertile, kuEx119-, and showed a Rol phenotype.

RNAi RNAi was carried out according to standard methods (Fire et al. 1998; Fraser et al. 2000). DAPI staining was according to Sulston and Hodgkin (1988).

Phylogenetic analysis Phylogenetic analysis was carried out with PAUP (Swafford 2000) using neighbor joining and maximum parsimony analysis with 500 bootstrap replicates. For each bootstrap, 10 heuristic searches of random addition sequence replicates were carried out.

Cyclin heat-shock strains Strains containing temperature-inducible cyclin E or cyclin A expression vectors were generated by injecting corresponding pPD49.78- and pPD49.83-derived constructs $(50 \mu \mathrm{g} / \mu \mathrm{L}$ each) along with sur-5::GFP $(100 \mu \mathrm{g} / \mu \mathrm{L})$ into unc-4, fzr-1 animals to obtain multiple stable extrachromosomal lines (cyclin A, fdEx1 and fdEx2; cyclin E, fdEx6 and $f d E x 7)$. Crossing to N2 generated $E x^{+}$lines in the wild-type background.

\section{Acknowledgments}

We thank the following people: Andy Fire for vectors; Yuji Kohara, Mike Krause, Paul Sternberg, and Karen Bennett for cDNAs and other clones; Joel Rothman, Eric Jorgensen, David Greenstein, Judith Kimble, and Jim McGhee for strains; Mike Krause, Ed Kipreos, Tin Tin Su, and Ann Rose for helpful discussions; Dan Starr and Amy Fluet for comments on the manuscript; and Scott Kelly for help with the phylogentic comparison. Some of the strains used in this work were provided by the Caenorhabditis Genetics Center, which is funded by the Na- tional Center for Research Resources of the NIH. D.S.F. was supported by an NRSA fellowship (F32GM20029-01). M.H. is an HHMI investigator. This work was supported by NIH grant GM37869.

The publication costs of this article were defrayed in part by payment of page charges. This article must therefore be hereby marked "advertisement" in accordance with 18 USC section 1734 solely to indicate this fact.

\section{References}

Blelloch, R., Anna-Arriola, S.S., Gao, D., Li, Y., Hodgkin, J. and Kimble, J. 1999. The gon-1 gene is required for gonadal morphogenesis in Caenorhabditis elegans. Dev. Biol. 216: 382393.

Boxem, M. and van den Heuvel, S. 2001. lin-35 Rb and cki-1 $\mathrm{Cip} /$ Kip cooperate in developmental regulation of G1 progression in C. elegans. Development 128: 4349-4359.

Brehm, A., Miska, E.A., McCance, D.J., Reid, J.L., Bannister, A.J., and Kouzarides, T. 1998. Retinoblastoma protein recruits histone deacetylase to repress transcription. Nature 391: 597-601.

Ceol, C.J. and Horvitz, H.R. 2001. dpl-1 DP and efl-1 E2F act with lin-35 $\mathrm{Rb}$ to antagonize Ras signaling in C. elegans vulval development. Mol. Cell 7: 461-473.

Chen, Z. and Han, M. 2001. C. elegans Rb, NuRD and Ras regulate lin-39-mediated cell fusion during vulval fates specification. Curr. Biol. 11: 1874-1879.

Clark, S.G., Lu, X., and Horvitz, H.R. 1994. The Caenorhabditis elegans locus lin-15, a negative regulator of a tyrosine kinase signaling pathway, encodes two different proteins. Genetics 137: 987-997.

Clarke, A.R., Maandag, E.R., van Roon, M., van der Lugt, N.M., van der Valk, M., Hooper, M.L., Berns, A., and te Riele, H. 1992. Requirement for a functional Rb-1 gene in murine development. Nature 359: 328-330.

Classon, M. and Dyson, N. 2001. p107 and p130: Versatile proteins with interesting pockets. Exp. Cell Res. 264: 135-147.

Cohen-Fix, O., Peters, J.M., Kirschner, M.W., and Koshland, D. 1996. Anaphase initiation in Saccharomyces cerevisiae is controlled by the APC-dependent degradation of the anaphase inhibitor Pds1p. Genes \& Dev. 10: 3081-3093.

Culotti, J.G., Von Ehrenstein, G., Culotti, M.R., and Russell, R.L. 1981. A second class of acetylcholinesterase-deficient mutants of the nematode Caenorhabditis elegans. Genetics 97: 281-305.

Davies, A.G., Spike, C.A., Shaw, J.E., and Herman, R.K. 1999. Functional overlap between the mec- 8 gene and five sym genes in Caenorhabditis elegans. Genetics 153: 117-134.

Dawson, I.A., Roth, S., and Artavanis-Tsakonas, S. 1995. The Drosophila cell cycle gene fizzy is required for normal degradation of cyclins $\mathrm{A}$ and $\mathrm{B}$ during mitosis and has homology to the CDC20 gene of Saccharomyces cerevisiae. J. Cell Biol. 129: 725-737.

DeGregori, J., Kowalik, T., and Nevins, J.R. 1995. Cellular targets for activation by the E2F1 transcription factor include DNA synthesis- and G1/S-regulatory genes. Mol. Cell. Biol. 15: 4215-4224.

de Nooij, J.C., Letendre, M.A., and Hariharan, I.K. 1996. A cyclin-dependent kinase inhibitor, Dacapo, is necessary for timely exit from the cell cycle during Drosophila embryogenesis. Cell 87: 1237-1247.

Deshaies R.J. 1999. SCF and Cullin/Ring H2-based ubiquitin ligases. Annu. Rev. Cell. Dev. Biol. 115: 435-467.

Dou, Q.P., Zhao, S., Levin, A.H., Wang, J., Helin, K., and Pardee, 
A.B. 1994. G1/S-regulated E2F-containing protein complexes bind to the mouse thymidine kinase gene promoter. J. Biol. Chem. 269: 1306-1313.

$\mathrm{Du}, \mathrm{W}$. and Dyson, N. 1999. The role of RBF in the introduction of G1 regulation during Drosophila embryogenesis. EMBO J. 18: 916-925.

Dunaief, J.L., Strober, B.E., Guha, S., Khavari, P.A., Alin, K., Luban, J., Begemann, M., Crabtree, G.R., and Goff, S.P. 1994. The retinoblastoma protein and BRG1 form a complex and cooperate to induce cell cycle arrest. Cell 79: 119-130.

Duronio, R.J. and O'Farrell, P.H. 1995. Developmental control of the G1 to S transition in Drosophila: Cyclin E is a limiting downstream target of E2F. Genes \& Dev. 9: 1456-1468.

Dyson, N. 1998. The regulation of E2F by pRB-family proteins. Genes \& Dev. 12: 2245-2262.

Fay, D.S. and Han, M. 2000. The synthetic multivulval genes of C. elegans: Functional redundancy, Ras-antagonism, and cell fate determination. Genesis 26: 279-284.

Ferguson, E.L. and Horvitz, H.R. 1989. The multivulva phenotype of certain Caenorhabditis elegans mutants results from defects in two functionally redundant pathways. Genetics 123: 109-121.

Ferguson, E.L., Sternberg, P.W., and Horvitz, H.R. 1987. A genetic pathway for the specification of the vulval cell lineages of Caenorhabditis elegans. Nature 326: 259-267.

Fire, A., Xu, S., Montgomery, M.K., Kostas, S.A., Driver, S.E., and Mello, C.C. 1998. Potent and specific genetic interference by double-stranded RNA in Caenorhabditis elegans. Nature 391: 806-811.

Flemington, E.K., Speck, S.H., and Kaelin, W.G., Jr. 1993. E2F1 -mediated transactivation is inhibited by complex formation with the retinoblastoma susceptibility gene product. Proc. Nat1. Acad. Sci. 90: 6914-6918.

Francis, R., Barton, M.K., Kimble, J., and Schedl, T. 1995. gld-1, a tumor suppressor gene required for oocyte development in Caenorhabditis elegans. Genetics 139: 579-606.

Fraser, A.G., Kamath, R.S., Zipperlen, P., Martinez-Campos, M., Sohrmann, M., and Ahringer, J. 2000. Functional genomic analysis of C. elegans chromosome I by systematic RNA interference. Nature 408: 325-330.

Fukushige, T., Hawkins, M.G., and McGhee, J.D. 1998. The GATA-factor elt-2 is essential for formation of the Caenorhabditis elegans intestine. Dev. Biol. 198: 286-302.

Gu, T., Orita, S., and Han, M. 1998. Caenorhabditis elegans SUR-5, a novel but conserved protein, negatively regulates LET-60 Ras activity during vulval induction. Mol. Cell. Biol. 18: 4556-4564.

Harbour, J.W. and Dean, D.C. 2000. The Rb/E2F pathway: Expanding roles and emerging paradigms. Genes \& Dev. 14: 2393-2409.

Hedgecock, E.M. and White, J.G. 1985. Polyploid tissues in the nematode Caenorhabditis elegans. Dev. Biol. 107: 128-133.

Helin, K., Harlow, E., and Fattaey, A. 1993. Inhibition of E2F-1 transactivation by direct binding of the retinoblastoma protein. Mol. Cell. Biol. 13: 6501-6508.

Hobert, O., D'Alberti, T., Liu, Y., and Ruvkun, G. 1998. Control of neural development and function in a thermoregulatory network by the LIM homeobox gene lin-11. I. Neurosci. 18: 2084-2096.

Hodgkin, J. 2001. What does a worm need with 20,000 genes? Genome Biol. 2: 2008.1-2008.4.

Hong, Y., Roy, R., and Ambros, V. 1998. Developmental regulation of a cyclin-dependent kinase inhibitor controls postembryonic cell cycle progression in Caenorhabditis elegans. Development 125: 3585-3597.

Huang, L.S., Tzou, P., and Sternberg, P.W. 1994. The lin-15 lo- cus encodes two negative regulators of Caenorhabditis elegans vulval development. Mol. Biol. Cell 5: 395-411.

Irniger, S. and Nasmyth, K. 1997. The anaphase-promoting complex is required in G1 arrested yeast cells to inhibit B-type cyclin accumulation and to prevent uncontrolled entry into S-phase. J. Cell Sci. 110: 1523-1531.

Irniger, S., Piatti, S., Michaelis, C., and Nasmyth, K. 1995. Genes involved in sister chromatid separation are needed for B-type cyclin proteolysis in budding yeast. Cell 81: 269-278.

Jacks, T., Fazeli, A., Schmitt, E.M., Bronson, R.T., Goodell, M.A., and Weinberg, R.A. 1992. Effects of an Rb mutation in the mouse. Nature 359: 295-300.

Johnson, C.D., Duckett, J.G., Culotti, J.G., Herman, R.K., Meneely, P.M., and Russell, R.L. 1981. An acetylcholinesterasedeficient mutant of the nematode Caenorhabditis elegans. Genetics 97: 261-279.

Kaelin, W.G., Jr. 1999. Functions of the retinoblastoma protein. Bioessays 21: 950-958.

King, R.W., Peters, J.M., Tugendreich, S., Rolfe, M., Hieter, P., and Kirschner, M.W. 1995. A 20S complex containing CDC27 and CDC16 catalyzes the mitosis-specific conjugation of ubiquitin to cyclin B. Cell 81: 279-288.

Kipreos, E.T., Lander, L.E., Wing, J.P., He, W.W., and Hedgecock, E.M. 1996. cul-1 is required for cell cycle exit in C. elegans and identifies a novel gene family. Cell 85: 829-839.

Kipreos, E.T., Gohel, S.P., and Hedgecock, E.M. 2000. The C. elegans F-box/WD-repeat protein LIN-23 functions to limit cell division during development. Development 127: 50715082.

Krause, M. and Hirsh, D. 1987. A trans-spliced leader sequence on actin mRNA in C. elegans. Cell 49: 753-761.

Lee, E.Y., Chang, C.Y., Hu, N., Wang, Y.C., Lai, C.C., Herrup, K., Lee, W.H., and Bradley, A. 1992. Mice deficient for Rb are nonviable and show defects in neurogenesis and haematopoiesis. Nature 359: 288-294.

Lipinski, M.M. and Jacks, T. 1999. The retinoblastoma gene family in differentiation and development. Oncogene 18: 7873-7882.

Lu, X. and Horvitz, H.R. 1998. lin-35 and lin-53, two genes that antagonize a C. elegans Ras pathway, encode proteins similar to $\mathrm{Rb}$ and its binding protein RbAp48. Cell 95: 981-991.

Luo, R.X., Postigo, A.A., and Dean, D.C. 1998. Rb interacts with histone deacetylase to repress transcription. Cell 92: 463473.

Magnaghi-Jaulin, L., Groisman, R., Naguibneva, I., Robin, P., Lorain, S., Le Villain, J.P., Troalen, F., Trouche, D., and Harel-Bellan, A. 1998. Retinoblastoma protein represses transcription by recruiting a histone deacetylase. Nature 391: 601-605.

McIntire, S.L., Reimer, R.J., Schuske, K., Edwards, R.H., and Jorgensen, E.M. 1997. Identification and characterization of the vesicular GABA transporter. Nature 389: 870-876.

Mello, C. and Fire, A. 1995. DNA transformation. Methods Cell Biol. 48: 451-482.

Morgan, D.O. 1999. Regulation of the APC and the exit from mitosis. Nat. Cell Biol. 1: E47-E53.

Nevins, J.R. 2001. The Rb/E2F pathway and cancer. Hum. Mol. Genet. 10: 699-703.

Nielsen, S.J., Schneider, R., Bauer, U.M., Bannister, A.J., Morrison, A., O'Carroll, D., Firestein, R., Cleary, M., Jenuwein, T., Herrera, R.E., et al. 2001. Rb targets histone H3 methylation and HP1 to promoters. Nature 412: 561-565.

Nowak, M.A., Boerlijst, M.C., Cooke, J., and Smith, J.M. 1997. Evolution of genetic redundancy. Nature 388: 167-171.

Ohtani, K., DeGregori, J., and Nevins, J.R. 1995. Regulation of the cyclin E gene by transcription factor E2F1. Proc. Nat1. 
Acad. Sci. 92: 12146-12150.

Park, M., and Krause, M.W. 1999. Regulation of postembryonic G(1) cell cycle progression in Caenorhabditis elegans by a cyclin D/CDK-like complex. Development 126: 4849-4860.

Schulze, A., Zerfass, K., Spitkovsky, D., Middendorp, S., Berges, J., Helin, K., Jansen-Durr, P., and Henglein, B. 1995. Cell cycle regulation of the cyclin A gene promoter is mediated by a variant E2F site. Proc. Natl. Acad. Sci. 92: 11264-11268.

Schwab, M., Lutum, A.S., and Seufert, W. 1997. Yeast Hct1 is a regulator of Clb2 cyclin proteolysis. Cell 90: 683-693.

Shi, Y. and Mello, C. 1998. A CBP/p300 homolog specifies multiple differentiation pathways in Caenorhabditis elegans. Genes \& Dev. 12: 943-955.

Sigrist, S.J. and Lehner, C.F. 1997. Drosophila fizzy-related down-regulates mitotic cyclins and is required for cell proliferation arrest and entry into endocycles. Cell 90: 671-681.

Sigrist, S., Jacobs, H., Stratmann, R., and Lehner, C.F. 1995. Exit from mitosis is regulated by Drosophila fizzy and the sequential destruction of cyclins $\mathrm{A}, \mathrm{B}$ and $\mathrm{B} 3$. EMBO $J$. 14: 4827-4838.

Smith, V., Chou, K.N., Lashkari, D., Botstein, D., and Brown, P.O. 1996. Functional analysis of the genes of yeast chromosome V by genetic footprinting. Science 274: 2069-2074.

Solari, F. and Ahringer, J. 2000. NURD-complex genes antagonise Ras-induced vulval development in Caenorhabditis elegans. Curr. Biol. 10: 223-226.

Sprenger, F., Yakubovich, N., and O'Farrell, P.H. 1997. S-phase function of Drosophila cyclin A and its downregulation in G1 phase. Curr. Biol. 7: 488-499.

Sudakin, V., Ganoth, D., Dahan, A., Heller, H., Hershko, J., Luca, F.C., Ruderman, J.V., and Hershko, A. 1995. The cyclosome, a large complex containing cyclin-selective ubiquitin ligase activity, targets cyclins for destruction at the end of mitosis. Mol. Biol. Cell 6: 185-197.

Sulston, J.E. and Hodgkin, J. 1988. Methods. In The nematode Caenorhabditis elegans (eds. W.B. Wood and the Community of C. elegans Researchers), pp. 587-606. Cold Spring Harbor Laboratory Press, Cold Spring Harbor, NY.

Sulston, J.E. and Horvitz, H.R. 1977. Post-embryonic cell lineages of the nematode, Caenorhabditis elegans. Dev. Biol. 56: $110-156$.

Swafford, D.L. 2000. PAUP phylogenetic analysis using parsimony (\& other methods), version 4. Sinauer Associates, Sunderland, MA.

Tautz, D. 2000. A genetic uncertainty problem. Trends Genet. 16: $475-477$.

Terns, R.M., Kroll-Conner, P., Zhu, J., Chung, S., and Rothman, J.H. 1997. A deficiency screen for zygotic loci required for establishment and patterning of the epidermis in Caenorhabditis elegans. Genetics 146: 185-206.

Thomas, J.H. and Horvitz, H.R. 1999. The C. elegans gene lin-36 acts cell autonomously in the lin-35 Rb pathway. Development 126: 3449-3459.

Visintin, R., Prinz, S., and Amon, A. 1997. CDC20 and CDH1: A family of substrate-specific activators of APC-dependent proteolysis. Science 278: 460-463.

von Zelewsky, T., Palladino, F., Brunschwig, K., Tobler, H., Hajnal, A., and Muller, F. 2000. The C. elegans Mi-2 chromatin-remodelling proteins function in vulval cell fate determination. Development 127: 5277-5284.

Wagner, A. 2000. Robustness against mutations in genetic networks of yeast. Nat. Genet. 24: 355-361.

Weintraub, S.J., Chow, K.N., Luo, R.X., Zhang, S.H., He, S., and Dean, D.C. 1995. Mechanism of active transcriptional repression by the retinoblastoma protein. Nature 375: 812-815.
Winzeler, E.A., Shoemaker, D.D., Astromoff, A., Liang, H., Anderson, K., Andre, B., Bangham, R., Benito, R., Boeke, J.D., Bussey, H., et al. 1999. Functional characterization of the $S$. cerevisiae genome by gene deletion and parallel analysis. Science 285: 901-906.

Zachariae, W. and Nasmyth, K. 1999. Whose end is destruction: Cell division and the anaphase-promoting complex. Genes \& Dev. 13: 2039-2058.

Zachariae, W., Schwab, M., Nasmyth, K., and Seufert, W. 1998. Control of cyclin ubiquitination by CDK-regulated binding of Hctl to the anaphase promoting complex. Science 282: 1721-1724.

Zhang, H.S., Gavin, M., Dahiya, A., Postigo, A.A., Ma, D., Luo, R.X., Harbour, J.W., and Dean, D.C. 2000. Exit from G1 and $\mathrm{S}$ phase of the cell cycle is regulated by repressor complexes containing HDAC-Rb-hSWI/SNF and Rb-hSWI/SNF. Cell 101: 79-89.

Zheng, L. and Lee, W.H. 2001. The retinoblastoma gene: A prototypic and multifunctional tumor suppressor. Exp. Cell Res. 264: 2-18.

Zur, A. and Brandeis, M. 2001. Securin degradation is mediated by $f z y$ and $f z r$, and is required for complete chromatid separation but not for cytokinesis. EMBO T. 20: 792-801. 


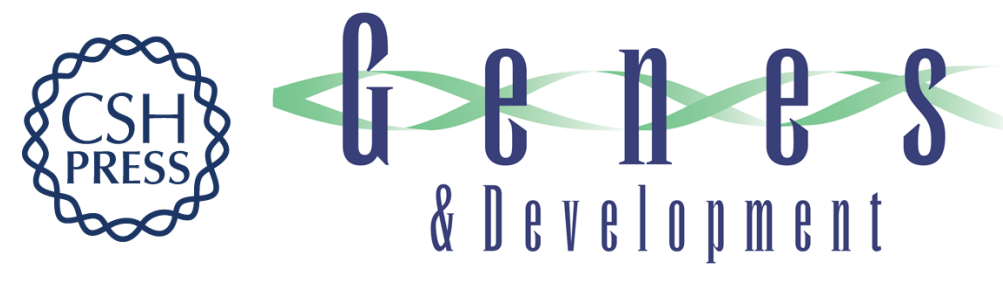

\section{fzr-1 and lin-35/Rb function redundantly to control cell proliferation in C. elegans as revealed by a nonbiased synthetic screen}

David S. Fay, Sean Keenan and Min Han

Genes Dev. 2002, 16:

Access the most recent version at doi:10.1101/gad.952302

References This article cites 81 articles, 37 of which can be accessed free at: http://genesdev.cshlp.org/content/16/4/503.full.htmI\#ref-list-1

License

Email Alerting

Receive free email alerts when new articles cite this article - sign up in the box at the top Service right corner of the article or click here.

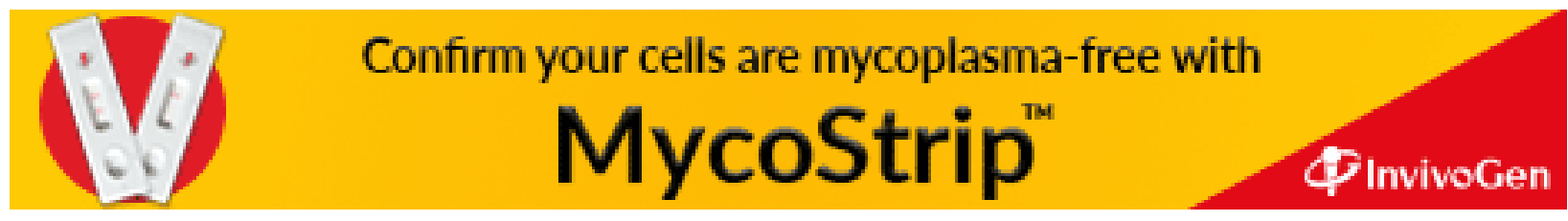

